\title{
A Flexible Semi-definite Programming Approach for Source Localization Problems
}

\author{
Frankie K.W. Chan, H. C. So. W.-K. Ma and Kenneth W. K. Lui
}

\begin{abstract}
In this paper, a new semi-definite programming approach is devised for approximating nonlinear estimation problems. The main idea is to include the noise components as parameters of interests, which increases the flexibility in the convex optimization formulation. Using the source localization as an illustration, we develop semi-definite relaxation (SDR) positioning algorithms using angle-of-arrival, time-of-arrival and time-difference-of-arrival measurements. Numerical examples are included to show the effectiveness of the proposed SDR approach.
\end{abstract}

\section{Index Terms}

Source localization, convex optimization, angle-of-arrival, time-of-arrival, time-different-of-arrival

\section{INTRODUCTION}

Finding the position of a target based on measurements from an array of spatially separated sensors has been one of the central problems in many fields such as radar, sonar [1], telecommunications [2], mobile communications [3] and wireless sensor networks [4]. Angle-of-arrival (AOA) [5], time-of-arrival (TOA) [6], time-difference-of-arrival (TDOA) [7], received signal strength (RSS) [8], and acoustic energy (AE) [9] of the emitted signal are commonly used measurements for source localization. Basically, AOAs are the source bearings relative to the receivers while TOAs, TDOAs, RSSs and AEs provide the distance information between the source and sensors. However, positioning is not a trivial task because these measurements are nonlinearly related to the source location. In this work, we focus on estimating the source position given the AOA, TOA or TDOA measurements. Without loss of generality, two-dimensional positioning is considered. AOA is the arrival angle of the emitted source signal observed at a receiver. From each AOA, we can draw a line of bearing (LOB) from the target to the receiver and the intersection of at least two LOBs will give the source location. On the other hand, in TOA-based positioning, the 
distance between the source and each receiver is determined from the one-way propagation time of the signal traveling between them. This provides a circle centered at the receiver on which the target must lie and it is clear that at least three receivers are needed to provide a unique solution. TDOA is the difference between two TOAs, which is employed when clock synchronization is not available at the source. That is, TDOAs correspond to the range-difference measurements and hyperbolic equations are constructed from them.

Nonlinear and linear [10] approaches are two conventional methodologies to solve for the position of the target. The first category deals with the nonlinear equations directly constructed from the AOA, TOA and TDOA measurements, which includes the nonlinear least squares (NLS) and maximum likelihood (ML) estimators. On the other hand, the second approach converts the nonlinear equations to be linear. Generally speaking, the former has superior estimation performance but there is no guarantee of obtaining the global solution because the corresponding cost functions are multi-modal. While the latter is computationally simple, its accuracy is often degraded by the linearization process especially under large noise conditions [11]-[12]. Convex optimization, particularly the semi-definite relaxation (SDR) method [13]-[15], is a more recent positioning approach which strikes a balance between nonlinear and linear methods, namely, high accuracy and global convergence. It starts from transforming the NLS or ML estimator to an equivalent constrained optimization problem and then relaxes the constraints so that a global solution can be obtained. Nevertheless, to the best of our knowledge, SDR-based positioning with solely AOA measurements has not been yet addressed in the literature.

The main contribution of this paper is to devise a new SDR formulation for positioning problems by considering the measurement noises as the parameters of interest. In doing so, the flexibility of expressing the NLS or ML estimation problems is highly increased. As a result, it is possible to obtain the SDR formulation for AOA-based positioning. Its development is provided in Section II and extension to receiver position uncertainty is also investigated. In Sections III and IV, we apply this new SDR formulation technique to TOA and TDOA measurements, respectively. In particular, we prove that in the uncorrelated noise scenario, our TOA-based position estimator is equivalent to a standard formulation [14]. Numerical examples for evaluating the performance of the proposed approach are included in Section V. Finally, conclusions are drawn in Section VI.

\section{LOCALIZATION WITH ANGLE-OF-ARRIVAL}

Line-of-sight transmission is assumed so that there is a direct propagation path between the source and each receiver. Let $\mathbf{x}=\left[\begin{array}{ll}x & y\end{array}\right]^{T}$ be the unknown source position and $\mathbf{x}_{l}=\left[x_{l} y_{l}\right]^{T}$ be the known coordinates 
of the $l$ th sensor, $l=1,2, \cdots, L$, where $L \geq 2$ is the number of receivers. In the following, we first address the basic AOA-based localization problem where the receiver positions are perfectly known and then extend the development when they are subject to uncertainty.

\section{A. Basic Algorithm}

The AOA measurements are modeled as:

$$
\phi_{l}=\tan ^{-1}\left(\frac{y_{l}-y}{x_{l}-x}\right)+q_{l}, \quad l=1,2, \cdots, L
$$

where $\left\{q_{l}\right\}$ are zero-mean Gaussian random variables with known covariance matrix $\mathbb{E}\left\{\mathbf{q q} \mathbf{q}^{T}\right\}=\mathbf{W}^{-1}$ with $\mathbf{q}=\left[\begin{array}{llll}q_{1} & q_{2} & \cdots & q_{L}\end{array}\right]^{T}$ and $\mathbb{E},{ }^{-1}$ and ${ }^{T}$ being the expectation, inverse and transpose operators, respectively. That is, $\mathbf{q} \sim \mathcal{N}\left(\mathbf{0}_{L \times 1}, \mathbf{W}^{-1}\right)$ where $\mathbf{0}_{M \times N}$ represents a $M \times N$ zero matrix. The task here is to find $\mathbf{x}$ given $\left\{\phi_{l}\right\}$ and $\left\{\mathbf{x}_{l}\right\}$. The ML estimator for $\mathbf{x}$ is

$$
\begin{array}{ll}
\underset{\tilde{\mathbf{x}}, \tilde{\boldsymbol{\psi}}}{\operatorname{minimize}} & (\tilde{\boldsymbol{\psi}}-\boldsymbol{\phi})^{T} \mathbf{W}(\tilde{\boldsymbol{\psi}}-\boldsymbol{\phi}) \\
\text { subject to } & \tilde{\boldsymbol{\psi}}=\left[\begin{array}{llll}
\tan ^{-1}\left(\frac{y_{1}-\tilde{y}}{x_{1}-\tilde{x}}\right) & \tan ^{-1}\left(\frac{y_{2}-\tilde{y}}{x_{2}-\tilde{x}}\right) & \cdots & \tan ^{-1}\left(\frac{y_{L}-\tilde{y}}{x_{L}-\tilde{x}}\right)
\end{array}\right]^{T}
\end{array}
$$

where $\phi=\left[\begin{array}{llll}\phi_{1} & \phi_{2} & \cdots & \phi_{L}\end{array}\right]^{T}$ and $\tilde{\boldsymbol{\psi}}$ represents the variable for $\boldsymbol{\psi}$. The main novelty in devising the new SDR formulation is to include the noises $\left\{q_{l}\right\}$ as variables in the ML cost function by expressing (2) as the following constrained optimization problem:

$$
\begin{array}{ll}
\underset{\tilde{\mathbf{x}}, \tilde{\mathbf{q}}, \tilde{\boldsymbol{\psi}}}{\operatorname{minimize}} & \tilde{\mathbf{q}}^{T} \mathbf{W} \tilde{\mathbf{q}} \\
\text { subject to } & \tilde{\mathbf{q}}=\boldsymbol{\phi}-\tilde{\boldsymbol{\psi}} \\
& \tilde{\boldsymbol{\psi}}=\left[\begin{array}{lllll}
\tan ^{-1}\left(\frac{y_{1}-\tilde{y}}{x_{1}-\tilde{x}}\right) & \tan ^{-1}\left(\frac{y_{2}-\tilde{y}}{x_{2}-\tilde{x}}\right) & \cdots & \tan ^{-1}\left(\frac{y_{L}-\tilde{y}}{x_{L}-\tilde{x}}\right)
\end{array}\right]^{T}
\end{array}
$$

Taking tangent on both sides of the first constraint in (3) and applying the difference formula for tangent, we obtain

$$
\begin{gathered}
\tan (\tilde{\mathbf{q}})=\tan (\boldsymbol{\phi}-\tilde{\boldsymbol{\psi}}) \\
\Rightarrow \tan (\tilde{\mathbf{q}})+\operatorname{diag}(\tan (\tilde{\boldsymbol{\psi}})) \operatorname{diag}(\tan (\boldsymbol{\phi})) \tan (\tilde{\mathbf{q}})=\tan (\boldsymbol{\phi})-\tan (\tilde{\boldsymbol{\psi}}) \\
\Rightarrow \operatorname{diag}\left(\mathbf{a}-\tilde{x} \mathbf{1}_{L}\right) \tan (\tilde{\mathbf{q}})+\operatorname{diag}\left(\mathbf{b}-\tilde{y} \mathbf{1}_{L}\right) \operatorname{diag}(\tan (\boldsymbol{\phi})) \tan (\tilde{\mathbf{q}})=\operatorname{diag}\left(\mathbf{a}-\tilde{x} \mathbf{1}_{L}\right) \tan (\boldsymbol{\phi})-\mathbf{b}+\tilde{y} \mathbf{1}_{L}
\end{gathered}
$$

where $\mathbf{a}=\left[\begin{array}{llll}x_{1} & x_{2} & \cdots & x_{L}\end{array}\right]^{T}, \mathbf{b}=\left[\begin{array}{llll}y_{1} & y_{2} & \cdots & y_{L}\end{array}\right]^{T}$ and $\mathbf{1}_{L}$ is a column vector of length $L$ with all entries equal one. Here, diag is the diagonal matrix operator and $\tan (\tilde{\mathbf{q}})$ corresponds to element-wise 
operation. Rearranging (4) yields

$$
\begin{aligned}
& \operatorname{diag}(\mathbf{a}) \tan (\boldsymbol{\phi})-\mathbf{b}=(\operatorname{diag}(\mathbf{a})+\operatorname{diag}(\mathbf{b}) \operatorname{diag}(\tan (\phi))) \tan (\tilde{\mathbf{q}})+x \tan (\boldsymbol{\phi})- \\
& y \mathbf{1}_{L}-x \tan (\tilde{\mathbf{q}})-y \operatorname{diag}(\tan (\boldsymbol{\phi})) \tan (\tilde{\mathbf{q}})
\end{aligned}
$$

Denoting $\tilde{\mathbf{p}}=\left[\tan (\tilde{\mathbf{q}})^{T} \quad \tilde{x} \quad \tilde{y}\right]^{T}$ and $\tilde{\mathbf{P}}=\tilde{\mathbf{p}} \tilde{\mathbf{p}}^{T}$, (5) can be expressed as

$$
\mathbf{y}=\mathbf{A} \tilde{\boldsymbol{\theta}}
$$

where

$$
\begin{aligned}
\mathbf{y} & =\operatorname{diag}(\mathbf{a}) \tan (\boldsymbol{\phi})-\mathbf{b} \\
\mathbf{A} & =\left[\begin{array}{lllll}
\operatorname{diag}(\mathbf{a})+\operatorname{diag}(\mathbf{b}) \operatorname{diag}(\tan (\boldsymbol{\phi})) & \tan (\boldsymbol{\phi}) & -\mathbf{1}_{L} & -\mathbf{I}_{L} & -\operatorname{diag}(\tan (\boldsymbol{\phi}))
\end{array}\right] \\
\tilde{\boldsymbol{\theta}} & =\left[\begin{array}{lll}
\tilde{\mathbf{p}}^{T} & \tilde{\mathbf{P}}_{1: L, L+1}^{T} & \tilde{\mathbf{P}}_{1: L: L+2}^{T}
\end{array}\right]^{T}
\end{aligned}
$$

with $\mathbf{I}_{L}$ and $\tilde{\mathbf{P}}_{i: m, j: n}$ being the $L \times L$ identity matrix and sub-matrix of $\tilde{\mathbf{P}}$ constructed from its $(i, j)$ to $(m, n)$ entries, respectively. Assuming that $\tilde{q}_{l}$ is sufficiently small, we have $\mathbf{P}_{l, l}=\tan ^{2}\left(q_{l}\right) \approx q_{l}^{2}$, $l=1,2, \cdots, L$. The optimization problem for AOA-based localization in (3) is then approximated as:

$$
\begin{array}{cl}
\underset{\tilde{\boldsymbol{\theta}}, \tilde{\mathbf{P}}}{\operatorname{minimize}} & \operatorname{tr}\left(\mathbf{W} \tilde{\mathbf{P}}_{1: L, 1: L}\right) \\
\text { subject to } & \mathbf{y}=\mathbf{A} \tilde{\boldsymbol{\theta}} \\
& \tilde{\mathbf{P}}=\tilde{\mathbf{p}} \tilde{\mathbf{p}}^{T}
\end{array}
$$

where $\operatorname{tr}$ denotes the trace operator. It is worth noting that although $\mathbf{y}=\mathbf{A} \tilde{\boldsymbol{\theta}}$ is underdetermined, unique solution is obtained through minimizing the objective function. Adopting the SDR approach, we relax (10) as a convex optimization problem as:

$$
\begin{array}{cl}
\underset{\tilde{\boldsymbol{\theta}}, \tilde{\mathbf{P}}}{\operatorname{minimize}} & \operatorname{tr}\left(\mathbf{W} \tilde{\mathbf{P}}_{1: L, 1: L}\right) \\
\text { subject to } & \mathbf{y}=\mathbf{A} \tilde{\boldsymbol{\theta}} \\
& {\left[\begin{array}{cc}
\tilde{\mathbf{P}} & \tilde{\mathbf{p}} \\
\tilde{\mathbf{p}}^{T} & 1
\end{array}\right] \succeq \mathbf{0}_{(L+3) \times(L+3)}}
\end{array}
$$

where $\succeq$ corresponds to the positive semi-definite operator.

\section{B. Extension to Receiver Position Uncertainty}

In practice, the receiver positions may not be known perfectly [15] and treating them to be exact may lead to degradation of estimation accuracy [16]-[17]. In this section, we take the receiver position 
uncertainty into account in the algorithm development. First, the erroneous receiver positions are modeled as:

$$
\begin{aligned}
& z_{l, 1}=x_{l}+u_{l, 1} \\
& z_{l, 2}=y_{l}+u_{l, 2}
\end{aligned}
$$

where $u_{l, 1}$ and $u_{l, 2}$ are the zero-mean disturbances in the $l$ th receiver position and $\mathbf{u}_{l} \sim \mathcal{N}\left(\mathbf{0}_{2 \times 1}, \Phi_{l}\right)$ with $\mathbf{u}_{l}=\left[\begin{array}{ll}u_{l, 1} & u_{l, 2}\end{array}\right]^{T}$ and $\boldsymbol{\Phi}_{l}$ is the covariance matrix for $\mathbf{u}_{l}$ which is assumed known. The AOA measurements are:

$$
\phi_{l}=\tan ^{-1}\left(\frac{z_{l, 2}-u_{l, 2}-y}{z_{l, 1}-u_{l, 1}-x}\right)+q_{l}, \quad l=1,2, \cdots, L
$$

Extending (2), the ML estimator for $\mathbf{x}$ is now:

$$
\begin{array}{ll}
\operatorname{minimize}_{\tilde{\boldsymbol{\psi}}, \tilde{\mathbf{x}}, \tilde{\mathbf{u}}_{1} \cdots \tilde{\mathbf{u}}_{L}} & (\tilde{\boldsymbol{\psi}}-\boldsymbol{\phi})^{T} \mathbf{W}(\tilde{\boldsymbol{\psi}}-\boldsymbol{\phi})+\sum_{l=1}^{L} \tilde{\mathbf{u}}_{l}^{T} \boldsymbol{\Phi}_{l}^{-1} \tilde{\mathbf{u}}_{l} \\
\text { subject to } & \tilde{\boldsymbol{\psi}}=\left[\begin{array}{llll}
\tan ^{-1}\left(\frac{z_{1,2}-\tilde{u}_{1,2}-\tilde{y}}{z_{1,1}-\tilde{u}_{1,1}-\tilde{x}}\right) & \tan ^{-1}\left(\frac{z_{2,2}-\tilde{u}_{2,2}-\tilde{y}}{z_{2,1}-\tilde{u}_{2,1}-\tilde{x}}\right) & \cdots & \tan ^{-1}\left(\frac{z_{L, 2}-\tilde{u}_{L, 2}-\tilde{y}}{z_{L, 1}-\tilde{u}_{L, 1}-\tilde{x}}\right)
\end{array}\right]^{T}
\end{array}
$$

Similar to (3), we reexpress the objective function in (15) as:

$$
\begin{aligned}
& \operatorname{minimize}_{\tilde{\boldsymbol{\psi}}, \tilde{\mathbf{x}}, \tilde{\mathbf{u}}_{1} \cdots \tilde{\mathbf{u}}_{L}, \tilde{\mathbf{q}}} \tilde{\mathbf{q}}^{T} \mathbf{W} \tilde{\mathbf{q}}+\sum_{l=1}^{L} \tilde{\mathbf{u}}_{l}^{T} \boldsymbol{\Phi}_{l}^{-1} \tilde{\mathbf{u}}_{l} \\
& \text { subject to } \tilde{\mathbf{q}}=\phi-\tilde{\boldsymbol{\psi}}
\end{aligned}
$$

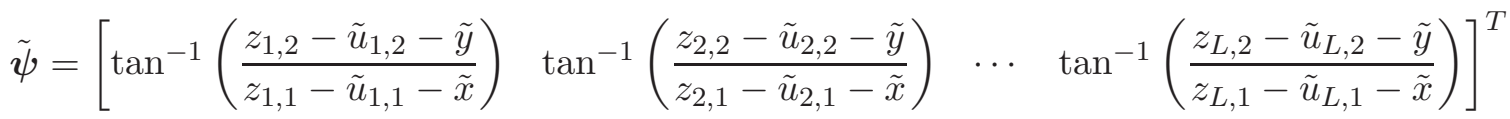

Following (4)-(5), we obtain from the first constraint in (16):

$$
\begin{gathered}
\tan (\tilde{\mathbf{q}})=\tan (\boldsymbol{\phi}-\tilde{\boldsymbol{\psi}}) \\
\Rightarrow \tan (\tilde{\mathbf{q}})+\operatorname{diag}(\tan (\tilde{\boldsymbol{\psi}})) \operatorname{diag}(\tan (\boldsymbol{\phi})) \tan (\tilde{\mathbf{q}})=\tan (\boldsymbol{\phi})-\tan (\tilde{\boldsymbol{\psi}}) \\
\Rightarrow \operatorname{diag}\left(\mathbf{z}_{1}-\tilde{\mathbf{u}}_{1}-\tilde{x} \mathbf{1}_{L}\right) \tan (\tilde{\mathbf{q}})+\operatorname{diag}\left(\mathbf{z}_{2}-\tilde{\mathbf{u}}_{2}-\tilde{y} \mathbf{1}_{L}\right) \operatorname{diag}(\tan (\boldsymbol{\phi})) \tan (\tilde{\mathbf{q}})= \\
\operatorname{diag}\left(\mathbf{z}_{1}-\tilde{\mathbf{u}}_{1}-\tilde{x} \mathbf{1}_{L}\right) \tan (\boldsymbol{\phi})-\mathbf{z}_{2}+\tilde{\mathbf{u}}_{2}+\tilde{y} \mathbf{1}_{L} \\
\Rightarrow \operatorname{diag}\left(\mathbf{z}_{1}\right) \tan (\boldsymbol{\phi})-\mathbf{z}_{2}=\left(\operatorname{diag}\left(\mathbf{z}_{1}\right)+\operatorname{diag}\left(\mathbf{z}_{2}\right) \operatorname{diag}(\tan (\boldsymbol{\phi}))\right) \tan (\tilde{\mathbf{q}})+\tilde{x} \tan (\boldsymbol{\phi})-\tilde{y} \mathbf{1}_{L}- \\
\tilde{x} \tan (\tilde{\mathbf{q}})-\tilde{y} \operatorname{diag}(\tan (\boldsymbol{\phi})) \tan (\tilde{\mathbf{q}})+\operatorname{diag}\left(\tilde{\mathbf{u}}_{1}\right) \tan (\boldsymbol{\phi})- \\
\operatorname{diag}\left(\tilde{\mathbf{u}}_{1}\right) \tan (\tilde{\mathbf{q}})-\operatorname{diag}\left(\tilde{\mathbf{u}}_{2}\right) \operatorname{diag}(\tan (\boldsymbol{\phi})) \tan (\tilde{\mathbf{q}})-\tilde{\mathbf{u}}_{2}
\end{gathered}
$$


where $\mathbf{z}_{1}=\left[\begin{array}{llll}z_{1,1} & z_{2,1} & \cdots & z_{L, 1}\end{array}\right]^{T}, \mathbf{z}_{2}=\left[\begin{array}{llll}z_{1,2} & z_{2,2} & \cdots & z_{L, 2}\end{array}\right]^{T}, \tilde{\mathbf{u}}_{1}=\left[\begin{array}{llll}\tilde{u}_{1,1} & \tilde{u}_{2,1} & \cdots & \tilde{u}_{L, 1}\end{array}\right]^{T}$ and $\tilde{\mathbf{u}}_{2}=\left[\begin{array}{llll}\tilde{u}_{1,2} & \tilde{u}_{2,2} & \cdots & \tilde{u}_{L, 2}\end{array}\right]^{T}$.

Denoting $\tilde{\mathbf{p}}=\left[\tan (\tilde{\mathbf{q}})^{T} \tilde{x} \quad \tilde{y} \quad \tilde{\mathbf{u}}_{1}^{T} \tilde{\mathbf{u}}_{2}^{T}\right]^{T}, \tilde{\mathbf{P}}=\tilde{\mathbf{p}} \tilde{\mathbf{p}}^{T}$ and $\mathbf{T}=\operatorname{diag}(\tan (\boldsymbol{\phi}))$, (17) can be written as

$$
\begin{array}{r}
\operatorname{diag}\left(\mathbf{z}_{1}\right) \tan (\boldsymbol{\phi})-\mathbf{z}_{2}=\left(\operatorname{diag}\left(\mathbf{z}_{1}\right)+\operatorname{diag}\left(\mathbf{z}_{2}\right) \mathbf{T}\right) \tan (\tilde{\mathbf{q}})+\tilde{x} \tan (\boldsymbol{\phi})-\tilde{y} \mathbf{1}_{L}-\tilde{\mathbf{P}}_{1: L, L+1}-\mathbf{T} \tilde{\mathbf{P}}_{1: L, L+2}+ \\
\mathbf{T} \tilde{\mathbf{p}}_{L+3: 2 L+2}-\operatorname{diag}\left(\tilde{\mathbf{P}}_{1: L, L+3: 2 L+2}\right)-\mathbf{T} \operatorname{diag}\left(\tilde{\mathbf{P}}_{1: L, 2 L+3: 3 L+2}\right)-\tilde{\mathbf{p}}_{2 L+3: 3 L+2}
\end{array}
$$

More compactly, we have

where

$$
\begin{aligned}
& \mathbf{y}=\mathbf{A} \tilde{\boldsymbol{\theta}} \\
& \mathbf{y}=\operatorname{diag}\left(\mathbf{z}_{1}\right) \tan (\boldsymbol{\phi})-\mathbf{z}_{2} \\
& \mathbf{A}=\left[\begin{array}{lllll}
\operatorname{diag}\left(\mathbf{z}_{1}\right)+\operatorname{diag}\left(\mathbf{z}_{2}\right) \mathbf{T} & \tan (\boldsymbol{\phi}) & -\mathbf{1}_{L} & -\mathbf{I}_{L} & -\mathbf{T}
\end{array}\right] \\
& \tilde{\boldsymbol{\theta}}=\left[\begin{array}{lll}
\tan (\tilde{\mathbf{q}})^{T} & \tilde{\mathbf{s}}^{T} & \tilde{\mathbf{t}}^{T}
\end{array}\right]^{T} \\
& \tilde{\mathbf{s}}=\tilde{\mathbf{P}}_{1: L, L+1}^{T}+\operatorname{diag}\left(\tilde{\mathbf{P}}_{1: L, L+3: 2 L+2}\right)^{T}+\tilde{\mathbf{p}}_{2 L+3: 3 L+2}^{T} \\
& \tilde{\mathbf{t}}=\tilde{\mathbf{P}}_{1: L: L+2}^{T}-\tilde{\mathbf{p}}_{L+3: 2 L+2}^{T}+\operatorname{diag}\left(\tilde{\mathbf{P}}_{1: L, 2 L+3: 3 L+2}\right)^{T}
\end{aligned}
$$

On the other hand, we rewrite the second term in the objective function of (15) as

where

$$
\tilde{\mathbf{H}}_{l}=\left[\begin{array}{cc}
\sum_{l=1}^{L} \tilde{\mathbf{P}}_{l}^{T} \boldsymbol{\Phi}_{l}^{-1} \tilde{\mathbf{u}}_{l}=\sum_{l=1}^{L} \operatorname{tr}\left(\boldsymbol{\Phi}_{l}^{-1} \tilde{\mathbf{H}}_{l}\right) \\
\tilde{\mathbf{P}}_{2 L+2+l, L+2+l} & \tilde{\mathbf{P}}_{L+2+l, 2 L+2+l}^{l=1} \\
\tilde{\mathbf{P}}_{2 L+2+l, 2 L+2+l}
\end{array}\right]
$$

Considering sufficiently small noise condition, the optimization problem for AOA-based localization with receiver position uncertainty in (15) is relaxed and approximated as

$$
\begin{aligned}
& \underset{\tilde{\boldsymbol{\theta}}, \tilde{\mathbf{P}}}{\operatorname{minimize}} \operatorname{tr}\left(\mathbf{W} \tilde{\mathbf{P}}_{1: L, 1: L}\right)+\sum_{l=1}^{L} \operatorname{tr}\left(\boldsymbol{\Phi}_{l}^{-1} \tilde{\mathbf{H}}_{l}\right) \\
& \text { subject to } \mathbf{y}=\mathbf{A} \tilde{\boldsymbol{\theta}} \\
& {\left[\begin{array}{cc}
\tilde{\mathbf{P}} & \tilde{\mathbf{p}} \\
\tilde{\mathbf{p}}^{T} & 1
\end{array}\right] \succeq \mathbf{0}_{(3 L+3) \times(3 L+3)}}
\end{aligned}
$$

\section{LOCALIZATION WITH TIME-OF-ARRIVAL}

The distance between the source and receiver is obtained by multiplying the TOA measurement by the propagation speed of the traveling signal. With $L \geq 3$ receivers, the position of the source can be determined. In the following, we first present the estimator for TOA-based localization in uncorrelated noises, which will be shown to be equivalent to a conventional SDR solution. Extensions to correlated noises and receiver position uncertainty are also included. 


\section{A. Basic Algorithm}

The range measurements are obtained by multiplying the TOAs with the known propagation speed, which are modeled as:

$$
r_{l}=d_{l}+q_{l}, \quad l=1,2, \cdots, L
$$

where $d_{l}$ represents the distance between the source and the $l$ th sensor and has the form of:

$$
d_{l}=\left\|\mathbf{x}-\mathbf{x}_{l}\right\|_{2}=\sqrt{\left(x-x_{l}\right)^{2}+\left(y-y_{l}\right)^{2}}, \quad l=1,2, \cdots, L
$$

with $\|\quad\|_{2}$ being the 2-norm operator. The $\left\{q_{l}\right\}$ denote the additive uncorrelated errors and each of them is modeled as $q_{l} \sim \mathcal{N}\left(0, \sigma_{l}^{2}\right)$, that is, $q_{l}$ is a zero-mean Gaussian process with known variance $\sigma_{l}^{2}$. The task here is to find $\mathbf{x}$ given $\left\{r_{l}\right\},\left\{\mathbf{x}_{l}\right\}$ and $\left\{\sigma_{l}^{2}\right\}$. Apparently, the ML estimator for $\mathbf{x}$ is

$$
\underset{\tilde{\mathbf{x}}}{\operatorname{minimize}} \sum_{l=1}^{L} \frac{1}{\sigma_{l}^{2}}\left(r_{l}-\left\|\tilde{\mathbf{x}}-\mathbf{x}_{l}\right\|_{2}\right)^{2},
$$

where $\tilde{\mathbf{x}}$ represents the variable for $\mathbf{x}$.

Analogous to Section II, we express (30) as the following constrained optimization problem:

$$
\begin{array}{ll}
\underset{\tilde{\mathbf{x}},\left\{\tilde{q}_{i}\right\}}{\operatorname{minimize}} & \sum_{l=1}^{L} \frac{\tilde{q}_{l}^{2}}{\sigma_{l}^{2}} \\
\text { subject to } & r_{l}-\tilde{q}_{l}=\left\|\tilde{\mathbf{x}}-\mathbf{x}_{l}\right\|_{2}, \quad l=1,2, \cdots, L
\end{array}
$$

Each nonlinear constraint in (31) can be converted to a linear one by squaring both sides and introducing new variables, namely, $\tilde{R}=\tilde{\mathbf{x}}^{T} \tilde{\mathbf{x}}$ and $\tilde{p}_{l}=\tilde{q}_{l}^{2}, l=1,2, \cdots, L$ :

$$
\left(r_{l}-\tilde{q}_{l}\right)^{2}=\left\|\tilde{\mathbf{x}}-\mathbf{x}_{l}\right\|_{2}^{2} \Leftrightarrow r_{l}^{2}-\mathbf{x}_{l}^{T} \mathbf{x}_{l}=\tilde{R}-2 \mathbf{x}_{l}^{T} \tilde{\mathbf{x}}+2 \tilde{q}_{l} r_{l}-\tilde{p}_{l}, \quad l=1,2, \cdots, L
$$

Note that the constraints in (31) and (32) are generally equivalent because $\tilde{q}_{l}>r_{l}$ does not align with minimizing $\sum_{l=1}^{L} \tilde{q}_{l}^{2} / \sigma_{l}^{2}$. As a result, we can rewrite (31) as:

$$
\begin{array}{cl}
\underset{\tilde{\boldsymbol{\theta}}}{\operatorname{minimize}} & \sum_{l=1}^{L} \frac{\tilde{p}_{l}}{\sigma_{l}^{2}} \\
\text { subject to } & \mathbf{y}=\mathbf{A} \tilde{\boldsymbol{\theta}} \\
& \tilde{p}_{l}=\tilde{q}_{l}^{2}, \quad l=1,2, \cdots, L \\
& \tilde{R}=\tilde{\mathbf{x}}^{T} \tilde{\mathbf{x}}
\end{array}
$$

where

$$
\mathbf{y}=\left[\begin{array}{c}
r_{1}^{2}-\mathbf{x}_{1}^{T} \mathbf{x}_{1} \\
r_{2}^{2}-\mathbf{x}_{2}^{T} \mathbf{x}_{2} \\
\vdots \\
r_{L}^{2}-\mathbf{x}_{L}^{T} \mathbf{x}_{L}
\end{array}\right]
$$




$$
\begin{gathered}
\mathbf{A}=\left[\begin{array}{cccccccccc}
-2 \mathbf{x}_{1}^{T} & 1 & 2 r_{1} & 0 & \cdots & 0 & -1 & 0 & \cdots & 0 \\
-2 \mathbf{x}_{2}^{T} & 1 & 0 & 2 r_{2} & \cdots & 0 & 0 & -1 & \cdots & 0 \\
\vdots & \vdots & \vdots & \vdots & \ddots & \vdots & \vdots & \vdots & \ddots & \vdots \\
-2 \mathbf{x}_{L}^{T} & 1 & 0 & 0 & \cdots & 2 r_{L} & 0 & 0 & \cdots & -1
\end{array}\right] \\
\tilde{\boldsymbol{\theta}}=\left[\begin{array}{cccc}
\tilde{\mathbf{x}} & \tilde{R} & \tilde{\mathbf{q}} & \tilde{\mathbf{p}}
\end{array}\right]^{T}
\end{gathered}
$$

with $\tilde{\mathbf{q}}=\left[\tilde{q}_{1}, \tilde{q}_{2}, \cdots, \tilde{q}_{L}\right]^{T}$ and $\tilde{\mathbf{p}}=\left[\tilde{p}_{1}, \tilde{p}_{2}, \cdots, \tilde{p}_{L}\right]^{T}$. Adopting the SDR approach, we relax (33) as a convex optimization problem as:

$$
\begin{array}{cl}
\underset{\tilde{\boldsymbol{\theta}}}{\operatorname{minimize}} & \sum_{l=1}^{L} \frac{\tilde{p}_{l}}{\sigma_{l}^{2}} \\
\text { subject to } & \mathbf{y}=\mathbf{A} \tilde{\boldsymbol{\theta}} \\
& \tilde{p}_{l} \geq \tilde{q}_{l}^{2}, \quad l=1,2, \cdots, L \\
& \tilde{R} \geq \tilde{\mathbf{x}}^{T} \tilde{\mathbf{x}}
\end{array}
$$

Note that the constraints of $\tilde{p}_{l} \geq \tilde{q}_{l}^{2}, l=1,2, \cdots, L$, are tight. It is because $\tilde{p}_{l}$ and $\tilde{q}_{l}$ have opposite signs in $\mathbf{y}=\mathbf{A} \tilde{\boldsymbol{\theta}}$ and minimizing $\tilde{p}_{l}$ implies maximizing $\tilde{q}_{l}$ which results in $\tilde{p}_{l}=\tilde{q}_{l}^{2}$. That is, relaxation is only employed in $\tilde{R} \geq \tilde{\mathbf{x}}^{T} \tilde{\mathbf{x}}$.

\section{B. Connection to Traditional Formulation}

A traditional SDR formulation for TOA-based positioning is [14]

$$
\begin{array}{lll}
\underset{\left\{\tilde{\beta}_{l}\right\},\left\{\tilde{g}_{l}\right\}, \tilde{R}, \tilde{\mathbf{x}}}{\operatorname{minimize}} & \sum_{l=1}^{L} \frac{1}{\sigma_{l}^{2}}\left(r_{l}^{2}-2 r_{l} \tilde{g}_{l}+\tilde{\beta}_{l}\right) & \\
\text { subject to } & \tilde{\beta}_{l}=\tilde{R}-2 \mathbf{x}_{l}^{T} \tilde{\mathbf{x}}+\mathbf{x}_{l}^{T} \mathbf{x}_{l}, \quad l=1,2, \cdots, L \\
& \tilde{\beta}_{l} \geq \tilde{g}_{l}^{2}, & \\
& \tilde{R} \geq \tilde{\mathbf{x}}^{T} \tilde{\mathbf{x}} &
\end{array}
$$

where $\tilde{g}_{l}=\left\|\tilde{\mathbf{x}}-\mathbf{x}_{l}\right\|_{2}, l=1,2, \cdots, L$. According to the constraint of (31), we employ $\tilde{g}_{l}=r_{l}-\tilde{q}_{l}$ and $\tilde{\beta}_{l}=\tilde{g}_{l}^{2}$ to express the objective function in (38) as

$$
r_{l}^{2}-2 r_{l} \tilde{g}_{l}+\tilde{\beta}_{l}=\left(r_{l}-\tilde{g}_{l}\right)^{2}=\tilde{q}_{l}^{2}=\tilde{p}_{l}
$$

On the other hand, the first set of constraints in (37) can be written as

$$
r_{l}^{2}-2 r_{l} \tilde{q}_{l}+\tilde{p}_{l}=\tilde{R}-2 \mathbf{x}_{l}^{T} \tilde{\mathbf{x}}+\mathbf{x}_{l}^{T} \mathbf{x}_{l}, \quad l=1,2, \cdots, L
$$


which is equivalent to the $l$ th row of $\mathbf{y}=\mathbf{A} \boldsymbol{\theta}$ after simple manipulations. Furthermore, (39) can be expressed as

$$
\begin{aligned}
\tilde{p}_{l} & =r_{l}^{2}-2 d_{l} \tilde{g}_{l}+\tilde{\beta}_{l}+\tilde{g}_{l}^{2}-\tilde{g}_{l}^{2} \\
& =\left(r_{l}-\tilde{g}_{l}\right)^{2}+\tilde{\beta}_{l}-\tilde{g}_{l}^{2} \\
& =\tilde{q}_{l}^{2}+\tilde{\beta}_{l}-\tilde{g}_{l}^{2}
\end{aligned}
$$

which implies

$$
\tilde{p}_{l} \geq \tilde{q}_{l}^{2} \Longleftrightarrow \tilde{\beta}_{l} \geq \tilde{g}_{l}^{2}, \quad l=1,2, \cdots, L
$$

That is, the second set of constraints in (38) is the same as that in (37). The equivalence between the two SDR formulations in (38) and (37) is thus proved.

\section{Extension to Correlated Noises}

For colored $\left\{q_{l}\right\}$ with known noise covariance matrix $\mathbf{W}^{-1}$, we follow (33) to obtain the ML estimator for $\mathrm{x}$ :

$$
\begin{array}{cl}
\underset{\tilde{\boldsymbol{\theta}}, \tilde{\mathbf{Q}}}{\operatorname{minimize}} & \operatorname{tr}(\mathbf{W} \tilde{\mathbf{Q}}) \\
\text { subject to } & \mathbf{y}=\mathbf{A} \tilde{\boldsymbol{\theta}} \\
& \tilde{\mathbf{Q}}=\tilde{\mathbf{q}} \tilde{\mathbf{q}}^{T} \\
& \tilde{R}=\tilde{\mathbf{x}}^{T} \tilde{\mathbf{x}}
\end{array}
$$

where $\tilde{\boldsymbol{\theta}}=\left[\begin{array}{llll}\tilde{\mathbf{x}}^{T} & \tilde{R} & \tilde{\mathbf{q}}^{T} & \operatorname{diag}(\tilde{\mathbf{Q}})^{T}\end{array}\right]^{T}$. Note that the elements of $\tilde{\mathbf{p}}$ in previous sections is the same as the diagonal elements of $\tilde{\mathbf{Q}}$. Applying relaxation, the corresponding SDR formulation is then:

$$
\begin{array}{cl}
\underset{\tilde{\boldsymbol{\theta}}, \tilde{\mathbf{Q}}}{\operatorname{minimize}} & \operatorname{tr}(\mathbf{W} \tilde{\mathbf{Q}}) \\
\text { subject to } & \mathbf{y}=\mathbf{A} \tilde{\boldsymbol{\theta}} \\
& {\left[\begin{array}{cc}
\tilde{\mathbf{Q}} & \tilde{\mathbf{q}} \\
\tilde{\mathbf{q}}^{T} & 1
\end{array}\right] \succeq \mathbf{0}_{(L+1) \times(L+1)}} \\
& \tilde{R} \geq \tilde{\mathbf{x}}^{T} \tilde{\mathbf{x}}
\end{array}
$$

D. Extension to Receiver Position Uncertainty

When the receiver positions are subject to uncertainty, the observed coordinates are:

$$
\mathbf{z}_{l}=\mathbf{x}_{l}+\mathbf{u}_{l}, \quad l=1,2, \cdots, L
$$


where $\mathbf{z}_{l}$ is the noisy version of $\mathbf{x}_{l}$ and it is assumed that the disturbance is modeled as $\mathbf{u}_{l} \sim \mathcal{N}\left(\mathbf{0}_{2 \times 1}, \mathbf{\Phi}_{l}\right)$ where $\boldsymbol{\Phi}_{l}, l=1,2, \cdots, L$, are known. By combining (45) and (29), the distance measurements are now:

$$
r_{l}=\left\|\mathbf{x}-\mathbf{x}_{l}\right\|_{2}+q_{l}=\left\|\mathbf{x}-\left(\mathbf{z}_{l}-\mathbf{u}_{l}\right)\right\|_{2}+q_{l}, \quad l=1,2, \cdots, L
$$

The ML estimator for the position according to (46) is then

$$
\begin{array}{ll}
\underset{\left\{\tilde{q}_{l}\right\},\left\{\tilde{\mathbf{u}}_{l}\right\}, \tilde{\mathbf{x}}}{\operatorname{minimize}} & \sum_{l=1}^{L} \frac{\tilde{q}_{l}^{2}}{\sigma_{l}^{2}}+\sum_{l=1}^{L} \tilde{\mathbf{u}}_{l}^{T} \boldsymbol{\Phi}_{l}^{-1} \tilde{\mathbf{u}}_{l} \\
\text { subject to } & r_{l}-\tilde{q}_{l}=\left\|\tilde{\mathbf{x}}-\left(\mathbf{z}_{l}-\tilde{\mathbf{u}}_{l}\right)\right\|_{2}, \quad l=1,2, \cdots, L
\end{array}
$$

To convert (47) into a SDR problem, we first rewrite the second term in the objective function as

$$
\sum_{l=1}^{L} \tilde{\mathbf{u}}_{l}^{T} \boldsymbol{\Phi}_{l}^{-1} \tilde{\mathbf{u}}_{l}=\sum_{l=1}^{L} \operatorname{tr}\left(\boldsymbol{\Phi}_{l}^{-1} \tilde{\boldsymbol{\Xi}}_{l}\right), \quad l=1,2 \cdots, L
$$

where $\tilde{\Xi}_{l}=\tilde{\mathbf{u}}_{l} \tilde{\mathbf{u}}_{l}^{T}$. On the other hand, we linearize the constraints by squaring both sides to obtain:

$$
r_{l}^{2}-\mathbf{z}_{l}^{T} \mathbf{z}_{l}=\tilde{\mathbf{x}}^{T} \tilde{\mathbf{x}}-2 \tilde{\mathbf{x}}^{T} \mathbf{z}_{l}+2 \mathbf{x}^{T} \tilde{\mathbf{u}}_{l}+2 r_{l} \tilde{q}_{l}-\tilde{q}_{l}^{2}-2 \mathbf{z}_{l}^{T} \tilde{\mathbf{u}}_{l}+\tilde{\mathbf{u}}_{l}^{T} \tilde{\mathbf{u}}_{l}, \quad l=1,2, \cdots, L
$$

The second-order terms can be removed by introducing new variables. That is, we let $\tilde{\mathbf{Y}}=\tilde{\mathbf{X}}^{T} \tilde{\mathbf{X}}$ where $\tilde{\mathbf{X}}=\left[\tilde{\mathbf{u}}_{1}, \tilde{\mathbf{u}}_{2}, \cdots, \tilde{\mathbf{u}}_{L}, \tilde{\mathbf{x}}\right]$, which means $\tilde{\mathbf{Y}}_{k, l}=\tilde{\mathbf{u}}_{k}^{T} \tilde{\mathbf{u}}_{l}, 1 \leq k, l \leq L$ and $\tilde{\mathbf{Y}}_{L+1, L+1}=\tilde{\mathbf{x}}^{T} \tilde{\mathbf{x}}$. Note that $\boldsymbol{\Xi}_{l}$ and $\mathbf{Y}_{l, l}$ are related by

$$
\operatorname{tr}\left(\tilde{\Xi}_{l}\right)=\tilde{\mathbf{u}}_{l}^{T} \tilde{\mathbf{u}}_{l}=\tilde{\mathbf{Y}}_{l, l}, \quad l=1,2, \cdots, L
$$

Using (48)-(50), (47) is expressed as

$$
\begin{aligned}
& \underset{\tilde{\mathbf{X}}, \tilde{\mathbf{Y}},\left\{\tilde{p}_{l}\right\},\left\{\tilde{q}_{l}\right\},\left\{\tilde{\boldsymbol{\Xi}}_{l}\right\}}{\operatorname{minimize}} \sum_{l=1}^{L} \frac{\tilde{p}_{l}}{\sigma_{l}^{2}}+\operatorname{tr}\left(\boldsymbol{\Phi}_{l}^{-1} \tilde{\boldsymbol{\Xi}}_{l}\right) \\
& \text { subject to } \quad r_{l}^{2}-\mathbf{z}_{l}^{T} \mathbf{z}_{l}=2 r_{l} \tilde{q}_{l}-2 \mathbf{z}_{l}^{T} \tilde{\mathbf{u}}_{l}-2 \mathbf{z}_{l}^{T} \tilde{\mathbf{x}}+ \\
& -\tilde{p}_{l}+2 \tilde{\mathbf{Y}}_{l, L+1}+\tilde{\mathbf{Y}}_{l, l}+\tilde{\mathbf{Y}}_{L+1, L+1}, \quad l=1,2, \cdots, L \\
& \tilde{p}_{l}=\tilde{q}_{l}^{2}, \quad l=1,2, \cdots, L \\
& \operatorname{tr}\left(\tilde{\boldsymbol{\Xi}}_{l}\right)=\tilde{\mathbf{Y}}_{l, l}, \quad i=1,2, \cdots, L \\
& \tilde{\boldsymbol{\Xi}}_{l}=\tilde{\mathbf{u}}_{l} \tilde{\mathbf{u}}_{l}^{T}, \quad l=1,2, \cdots, L \\
& \tilde{\mathbf{Y}}=\tilde{\mathbf{X}}^{T} \tilde{\mathbf{X}}
\end{aligned}
$$


Writing the constraints of (51) in matrix form and applying relaxation, we have the following SDR formulation:

$$
\begin{aligned}
& \underset{\tilde{\mathbf{X}}, \tilde{\mathbf{Y}}, \tilde{\mathbf{p}}, \tilde{\mathbf{q}},\left\{\tilde{\boldsymbol{\Xi}}_{l}\right\}}{\operatorname{minimize}} \sum_{l=1}^{L} \frac{\tilde{p}_{l}}{\sigma_{l}^{2}}+\operatorname{tr}\left(\boldsymbol{\Phi}_{l}^{-1} \tilde{\boldsymbol{\Xi}}_{l}\right) \\
& \text { subject to } \mathbf{y}=\mathbf{A} \tilde{\boldsymbol{\theta}} \\
& \tilde{p}_{l} \geq \tilde{q}_{l}^{2}, \\
& l=1,2, \cdots, L \\
& \operatorname{tr}\left(\tilde{\Xi}_{l}\right)=\tilde{\mathbf{Y}}_{l, l}, \quad l=1,2, \cdots, L \\
& \begin{array}{l}
{\left[\begin{array}{cc}
\tilde{\boldsymbol{\Xi}}_{l} & \tilde{\mathbf{u}}_{l} \\
\tilde{\mathbf{u}}_{l}^{T} & 1
\end{array}\right] \succeq \mathbf{0}_{3 \times 3},} \\
{\left[\begin{array}{cc}
\tilde{\mathbf{Y}} & \tilde{\mathbf{X}}^{T} \\
\tilde{\mathbf{X}} & \mathbf{I}_{2}
\end{array}\right] \succeq \mathbf{0}_{(L+3) \times(L+3)}}
\end{array} \\
& \text { where } \quad \mathbf{y}=\left[\begin{array}{llll}
r_{1}^{2}-2 \mathbf{z}_{1}^{T} \mathbf{z}_{1} & r_{2}^{2}-2 \mathbf{z}_{2}^{T} \mathbf{z}_{2} & \cdots & r_{L}^{2}-\mathbf{z}_{L}^{T} \mathbf{z}_{L}
\end{array}\right]^{T} \\
& \mathbf{A}=\left[\begin{array}{llllllll}
2 \operatorname{diag}\left(r_{1}, \cdots, r_{L}\right) & -2 \operatorname{diag}\left(\mathbf{z}_{1}^{T}, \mathbf{z}_{2}^{T}, \cdots, \mathbf{z}_{L}^{T}\right) & -2 \mathbf{Z}^{T} & -\mathbf{I}_{L} & 2 \mathbf{I}_{L} & \mathbf{I}_{L} & \mathbf{1}_{L}
\end{array}\right]
\end{aligned}
$$

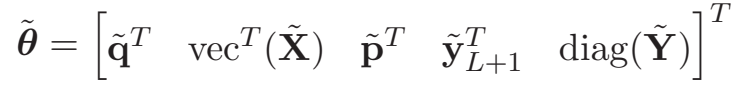

$$
\begin{aligned}
& \tilde{\mathbf{y}}_{L+1}=\left[\begin{array}{llll}
\tilde{\mathbf{Y}}_{1, L+1} & \tilde{\mathbf{Y}}_{2, L+1} & \cdots & \tilde{\mathbf{Y}}_{L, L+1}
\end{array}\right]^{T} \\
& \tilde{\mathbf{p}}=\left[\begin{array}{llll}
\tilde{p}_{1} & \tilde{p}_{2} & \cdots & \tilde{p}_{L}
\end{array}\right]^{T} \\
& \tilde{\mathbf{q}}=\left[\begin{array}{llll}
\tilde{q}_{1} & \tilde{q}_{2} & \cdots & \tilde{q}_{L}
\end{array}\right]^{T} \\
& \mathbf{Z}=\left[\begin{array}{llll}
\mathbf{z}_{1} & \mathbf{z}_{2} & \cdots & \mathbf{z}_{L}
\end{array}\right]
\end{aligned}
$$

\section{LOCALIZATION With TIME-DiffERENCE-OF-ARRIVAL}

Without loss of generality, let the first receiver be the reference. Based on the TDOA information, the range-difference measurement between the $l$ th and the first receivers, denoted by $r_{l, 1}$, is:

$$
r_{l, 1}=d_{l}-d_{1}+q_{l, 1}, \quad l=2,3, \cdots, L
$$

When the observed data at the receivers are processed optimally [18], the disturbances $\left\{q_{l, 1}\right\}$ can be modeled as $\left(q_{l}-q_{1}\right)$ where $\left\{q_{l}\right\}$ are uncorrelated zero-mean Gaussian processes with known variances $\left\{\sigma_{l}^{2}\right\}$. In TDOA-based positioning, $\mathbf{x}$ is estimated using $r_{l, 1}, l=2,3, \cdots, L, \mathbf{x}_{l}$ and $\sigma_{l}^{2}, l=1,2, \cdots, L$.

Rearranging and squaring both sides of (60), we have

$$
\begin{gathered}
\left(r_{l, 1}+\left\|\mathbf{x}-\mathbf{x}_{1}\right\|_{2}\right)^{2}=\left(\left\|\mathbf{x}-\mathbf{x}_{l}\right\|_{2}+q_{l}-q_{1}\right)^{2}, \quad l=2,3, \cdots, L \\
r_{l, 1}^{2}-2 \mathbf{x}_{1}^{T} \mathbf{x}+\mathbf{x}_{1}^{T} \mathbf{x}_{1}+2 r_{l, 1} d_{1}=\mathbf{x}_{l}^{T} \mathbf{x}_{l}-2 \mathbf{x}_{l}^{T} \mathbf{x}+\left(q_{l}-q_{1}\right)^{2}+2\left(q_{l}-q_{1}\right) d_{l}, \quad l=2,3, \cdots, L
\end{gathered}
$$


Substituting $d_{l}$ by $r_{l, 1}+d_{1}-\left(q_{l}-q_{1}\right)$ to reduce the number of variables, we obtain

$$
\begin{array}{r}
r_{l, 1}^{2}-\mathbf{x}_{l}^{T} \mathbf{x}_{l}+\mathbf{x}_{1}^{T} \mathbf{x}_{1}=2\left(\mathbf{x}_{1}^{T}-\mathbf{x}_{l}^{T}\right) \mathbf{x}-2 r_{l, 1} q_{1}+2 r_{l, 1} q_{l}-2 r_{l, 1} d_{1}-2 d_{1} q_{1}+2 d_{1} q_{l}-q_{1}^{2}-q_{l}^{2}+2 q_{l} q_{1} \\
l=2,3, \cdots, L \quad(62)
\end{array}
$$

which can be written in matrix form as

$$
\mathbf{y}=\mathbf{A} \boldsymbol{\theta}
$$

where $\quad \mathbf{y}=\left[\begin{array}{llll}r_{2,1}^{2}-\mathbf{x}_{2}^{T} \mathbf{x}_{2}+\mathbf{x}_{1}^{T} \mathbf{x}_{1} & r_{3,1}^{2}-\mathbf{x}_{3}^{T} \mathbf{x}_{3}+\mathbf{x}_{1}^{T} \mathbf{x}_{1} & \cdots & d_{L, 1}^{2}-\mathbf{x}_{L}^{T} \mathbf{x}_{L}+\mathbf{x}_{1}^{T} \mathbf{x}_{1}\end{array}\right]^{T}$

$$
\begin{aligned}
& \mathbf{A}=\left[\begin{array}{lllll}
2 \mathbf{X} & 2 \mathbf{P}_{r} & 2 \mathbf{P}_{+} & \mathbf{P}_{-} & 2 \mathbf{I}_{L-1}
\end{array}\right] \\
& \boldsymbol{\theta}=\left[\begin{array}{lllll}
\mathbf{x}^{T} & \mathbf{q}^{T} & \mathbf{Q}_{1: L, L+1}^{T} & \operatorname{diag}\left(\mathbf{Q}_{1: L, 1: L}\right) & \mathbf{Q}_{2: L, 1}^{T}
\end{array}\right]^{T} \\
& \mathbf{X}=\left[\begin{array}{llll}
\mathbf{x}_{1}-\mathbf{x}_{2} & \mathbf{x}_{1}-\mathbf{x}_{3} & \cdots & \mathbf{x}_{1}-\mathbf{x}_{L}
\end{array}\right] \\
& \mathbf{P}_{r}=\left[\begin{array}{lll}
-\mathbf{r} & \operatorname{diag}(\mathbf{r}) & -\mathbf{r}
\end{array}\right] \\
& \mathbf{r}=\left[\begin{array}{llll}
r_{2,1} & r_{3,1} & \cdots & r_{L, 1}
\end{array}\right]^{T} \\
& \mathbf{P}_{ \pm}=\left[\begin{array}{ll}
-\mathbf{1}_{L-1} & \pm \mathbf{I}_{L-1}
\end{array}\right] \\
& \mathbf{q}=\left[\begin{array}{lllll}
q_{1} & q_{2} & \cdots & q_{L} & d_{1}
\end{array}\right]^{T} \\
& \mathbf{Q}=\mathbf{q q}^{T}
\end{aligned}
$$

Note that $\mathbf{Q}_{L+1, L+1}$ is related to $\mathbf{x}$ as

$$
\mathbf{Q}_{L+1, L+1}=d_{1}^{2}=R-2 \mathbf{x}^{T} \mathbf{x}_{1}+\mathbf{x}_{1}^{T} \mathbf{x}_{1}
$$

where $R=\mathbf{x}^{T} \mathbf{x}$ is introduced to make the equation linear. Then, the optimization problem becomes

$$
\begin{array}{cl}
\underset{\tilde{\boldsymbol{\theta}}, \tilde{R}, \tilde{\mathbf{Q}}}{\operatorname{minimize}} & \operatorname{tr}\left(\mathbf{W} \tilde{\mathbf{Q}}_{1: L, 1: L}\right) \\
\text { subject to } & \mathbf{y}=\mathbf{A} \tilde{\boldsymbol{\theta}} \\
& \tilde{\mathbf{Q}}=\tilde{\mathbf{q}} \tilde{\mathbf{q}}^{T} \\
& \tilde{\mathbf{Q}}_{L+1, L+1}=\tilde{R}-2 \mathbf{x}_{1}^{T} \tilde{\mathbf{x}}+\mathbf{x}_{1}^{T} \mathbf{x}_{1} \\
& \tilde{R}=\tilde{\mathbf{x}}^{T} \tilde{\mathbf{x}}
\end{array}
$$

where $\mathbf{W}=\operatorname{diag}\left(1 / \sigma_{1}^{2}, 1 / \sigma_{2}^{2}, \cdots, 1 / \sigma_{L}^{2}\right)$. Relaxing the non-convex constraints in (74) gives the SDR 
formulation for TDOA-based positioning:

$$
\begin{array}{cl}
\underset{\tilde{\boldsymbol{\theta}}, \tilde{R}, \tilde{\mathbf{Q}}}{\operatorname{minimize}} & \operatorname{tr}\left(\mathbf{W} \tilde{\mathbf{Q}}_{1: L, 1: L}\right) \\
\text { subject to } & \mathbf{y}=\mathbf{A} \tilde{\boldsymbol{\theta}} \\
& \tilde{\mathbf{Q}} \succeq \tilde{\mathbf{q}} \tilde{\mathbf{q}}^{T}, \\
& \tilde{\mathbf{Q}}_{L+1, L+1}=\tilde{R}-2 \mathbf{x}_{1}^{T} \tilde{\mathbf{x}}+\mathbf{x}_{1}^{T} \mathbf{x}_{1}, \\
& \tilde{R} \geq \tilde{\mathbf{x}}^{T} \tilde{\mathbf{x}}
\end{array}
$$

When the receiver positions are not perfectly known, we have $\mathbf{x}_{l}=\mathbf{z}_{l}-\mathbf{u}_{l}$ according to (36). Substituting $d_{l}=\left\|\mathbf{x}-\mathbf{z}_{l}+\mathbf{u}_{l}\right\|$ into (61) yields

$$
\begin{aligned}
r_{l, 1}^{2}-2\left(\mathbf{z}_{l}+\mathbf{u}_{l}\right)^{T} \mathbf{x} & +\left(\mathbf{z}_{1}+\mathbf{u}_{1}\right)^{T}\left(\mathbf{z}_{1}+\mathbf{u}_{1}\right)+2 r_{l, 1} d_{1} \\
& =\left(\mathbf{z}_{l}+\mathbf{u}_{l}\right)^{T} \mathbf{x}_{l}-2\left(\mathbf{z}_{l}+\mathbf{u}_{l}\right)^{T} \mathbf{x}+\left(q_{l}-q_{1}\right)^{2}+2\left(q_{l}-q_{1}\right) d_{l}, \quad l=2,3, \cdots, L
\end{aligned}
$$

Expressing (76) in matrix form, we have $\mathbf{y}=\mathbf{A} \boldsymbol{\theta}$ where

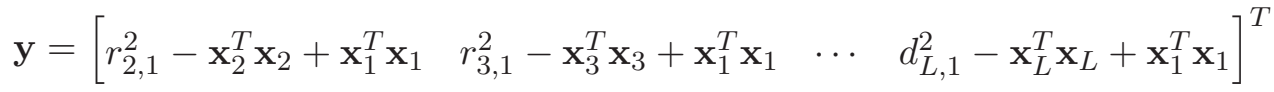

$$
\begin{aligned}
& \mathbf{A}=\left[\begin{array}{llllllllll}
2 \mathbf{P}_{r} & 2 \mathbf{X} & 2 \mathbf{1}_{L-1} \mathbf{x}_{1}^{T} & -2 \operatorname{diag}\left(\mathbf{x}_{2}^{T}, \cdots, \mathbf{x}_{L}^{T}\right) & 2 \mathbf{P}_{+} & \mathbf{P}_{-} & 2 \mathbf{I}_{L-1} & 2 \mathbf{P}_{+} & \mathbf{P}_{+}
\end{array}\right] \\
& \boldsymbol{\theta}=\left[\begin{array}{llllll}
\mathbf{q}^{T} & \mathbf{Q}_{1: L, 1: L}^{T} & \operatorname{diag}\left(\mathbf{Q}_{1: L, 1: L}\right) & \mathbf{Q}_{2: L, 1}^{T} & \mathbf{g}_{1}^{T} & \mathbf{g}_{2}^{T}
\end{array}\right]^{T} \\
& \mathbf{X}=\left[\begin{array}{llll}
\mathbf{x}_{1}-\mathbf{x}_{2} & \mathbf{x}_{1}-\mathbf{x}_{3} & \cdots & \mathbf{x}_{1}-\mathbf{x}_{L}
\end{array}\right] \\
& \mathbf{g}_{1}=\left[\begin{array}{llll}
\operatorname{tr}\left(\mathbf{Q}_{L+2: L+3, L+4: L+5}\right) & \operatorname{tr}\left(\mathbf{Q}_{L+2: L+3, L+6: L+7}\right) & \cdots & \operatorname{tr}\left(\mathbf{Q}_{L+2: L+3,3 L+2: 3 L+3}\right)
\end{array}\right]^{T} \\
& \mathbf{g}_{2}=\left[\begin{array}{llll}
\operatorname{tr}\left(\mathbf{Q}_{L+4: L+5, L+4: L+5}\right) & \operatorname{tr}\left(\mathbf{Q}_{L+6: L+7, L+6: L+7}\right) & \cdots & \operatorname{tr}\left(\mathbf{Q}_{3 L+2: 3 L+3,3 L+2: 3 L+3}\right)
\end{array}\right]^{T} \\
& \mathbf{q}=\left[\begin{array}{llllllll}
q_{1} & \cdots & q_{L} & d_{1} & \mathbf{x}^{T} & \mathbf{u}_{1}^{T} & \cdots & \mathbf{u}_{L}^{T}
\end{array}\right]^{T}
\end{aligned}
$$

The SDR formulation of (75) is modified as

$$
\begin{array}{ll}
\underset{\tilde{\boldsymbol{\theta}}, \tilde{\mathbf{Q}}}{\operatorname{minimize}} & \operatorname{tr}\left(\mathbf{W} \tilde{\mathbf{Q}}_{1: L, 1: L}\right)+\sum_{l=1}^{L} \operatorname{tr}\left(\boldsymbol{\Phi}_{l}^{-1} \tilde{\mathbf{Q}}_{L+l+3: L+l+4, L+l+3: L+l+4}\right) \\
\text { subject to } & \mathbf{y}=\mathbf{A} \tilde{\boldsymbol{\theta}}, \\
& \tilde{\mathbf{Q}} \succeq \tilde{\mathbf{q}} \tilde{\mathbf{q}}^{T}, \\
& \tilde{\mathbf{Q}}_{L+1, L+1}=\tilde{\mathbf{Q}}_{L+2, L+2}+\tilde{\mathbf{Q}}_{L+3, L+3}+\mathbf{z}_{1}^{T} \mathbf{z}_{1} \\
& +\tilde{\mathbf{Q}}_{L+4, L+4}+\tilde{\mathbf{Q}}_{L+5, L+5}-2 \mathbf{z}_{1}^{T} \tilde{\mathbf{q}}_{M+2: M+3} \\
& \\
& +2 \tilde{\mathbf{Q}}_{L+4, L+2}+2 \tilde{\mathbf{Q}}_{L+5, L+3}-2 \mathbf{z}_{1}^{T} \mathbf{q}_{L+4: L+5}
\end{array}
$$

where the last equality corresponds to $\tilde{R} \geq \tilde{\mathbf{x}}^{T} \tilde{\mathbf{x}}$. 


\section{Simulation Results}

Computer simulation has been conducted to evaluate the performance of the proposed SDR source localization methods. We utilize the MATLAB toolbox YALMIP [19] for the algorithm realization where the solver SDPT3 [20]-[21] is employed. Unless stated otherwise, we examine the geometry depicted in Figure 1 where the receivers are located at $(10,0),(5,5 \sqrt{3}),(-5,5 \sqrt{3}),(-10,0),(-5,-5 \sqrt{3})$ and $(5,-5 \sqrt{3})$ while the source position is located at $(-7,9)$. Note that the source is located outside the convex hull of the receivers. In the presence of colored noise, the corresponding covariance is assigned as a Toeplitz symmetric matrix with the first row being $\left[\begin{array}{llllll}10 & 9 & 8 & 7 & 6 & 5\end{array}\right] \sigma^{2}$ in AOA-based and TOA-based positioning. When the receiver positions are subject to errors, we assign $\mathbf{\Phi}_{l}=10^{0.1} \mathbf{I}_{2}, l=1,2, \cdots, L$. The mean square error (MSE) is employed as the performance measure and all the results are based on averages of 200 independent runs.

First, we investigate the performance of the proposed SDR approach for AOA-based localization. Comparisons with Cramér-Rao lower bound (CRLB) and [12] which is a linear least squares method, are also made. We consider correlated AOA measurements and the MSE performance versus $\sigma^{2}$ is plotted in Figure 2. We observe that the proposed SDR method is superior to [12] and its MSEs are close to the CRLB when $\sigma^{2} \leq-8 \mathrm{~dB}$. The average computational times per run are measured as $0.73 \mathrm{~s}$ and $5.2 \times 10^{-5} \mathrm{~s}$, respectively. Figure 3 shows the results of presence of uncertainty in the receiver positions. It is seen that the algorithm in [12] perform slightly better but both algorithms cannot attain the CRLB.

Second, TOA-based localization is addressed and we first compare the proposed scheme with the standard SDR algorithm [14] and CRLB in the uncorrelated noise scenarios. For ease of presentation but without loss of generality, we use $\sigma^{2}=\sigma_{l}^{2}, l=1,2, \cdots, L$. The results are shown in Figure 4 and we see that there is no obvious difference between the two methods, which aligns with our analysis, and their MSEs are close to the CRLB. Moreover, the average run times of the proposed and standard algorithms are $0.32 \mathrm{~s}$ and $0.33 \mathrm{~s}$, respectively, indicating that their complexity is similar. This test is repeated such that the source position is randomly chosen from a circle with center $(0,0)$ and radius 15 at each trial, and the results are plotted in Figure 5. The findings are similar to those of Figure 4. Figure 6 shows the results for the colored noise case and it is seen that the proposed algorithm outperforms [14]. Finally, the first test is repeated with receiver position uncertainty and the MSE performance is plotted in Figure 7. Both methods perform similarly and their MSEs are close to the CRLB for the whole range of $\sigma^{2}$.

Finally, the proposed SDR algorithm for TDOA-based localization is investigated and we first compare with [15] when the receiver positions are known exactly. The receiver with position $(10,0)$ is employed 
as the reference and the results are shown in Figure 8. It is seen that the performance of the SDR method degrades the CRLB by about $1.5 \mathrm{~dB}$ because the second constraint in (75) is not tight. However, it is superior to [15] when the noise power is larger than $-10 \mathrm{~dB}$. The computational times of the proposed SDR algorithm and [15] are $0.92 \mathrm{~s}$ and $0.78 \mathrm{~s}$, respectively. Figure 9 shows the MSEs when there is receiver position uncertainty and the results of [16] are also included. The performance of the proposed scheme is in between [15] and [16], and all cannot attain CRLB.

\section{CONCLUSION}

By including the noise components as the variables, a new semi-definite relaxation (SDR) formulation, which allows higher design flexibility, is devised. Based on the proposed approach, SDR algorithms for source localization using angle-of-arrival, time-of-arrival and time-difference-of-arrival measurements are developed. Simulation results show that the performance of the SDR schemes are close to the Cramér-Rao lower bound when the constraints are tight.

\section{REFERENCES}

[1] G.C. Carter, Ed., Coherence and Time Delay Estimation: An Applied Tutorial for Research, Development, Test, and Evaluation Engineers, New York: IEEE, 1993

[2] Y. Huang and J.Benesty, Eds., Audio Signal Processing for Next-Generation Multimedia Communication Systems, Kluwer Academic Publishers, 2004

[3] J.J. Caffery, Jr., Wireless Location in CDMA Cellular Radio Systems, Kluwer Academic Publishers, 2000

[4] M. Ilyas and I. Mahgoub, Handbook of Sensor Networks: Compact Wireless and Wired Sensing Systems, London, U.K.: CRC Press, 2005

[5] J.C. Chen, Y. Kung and R.E. Hudson, "Source localization and beamforming," IEEE Signal Processing Magazine, vol.19, no.2, pp.30-39, 2002

[6] K.W. Cheung, H.C. So, W.-K. Ma and Y.T. Chan, "Least squares algorithms for time-of-arrival based mobile location," IEEE Transactions on Signal Processing, vol.52, no.4, pp.1121-1128, Apr. 2004

[7] Y.T. Chan and K.C. Ho, "A simple and efficient estimator for hyperbolic location," IEEE Transactions on Signal Processing, vol.42, pp.1905-1915, Aug. 1994

[8] X. Li, "Collaborative localization with received signal strength in wireless sensor networks," IEEE Transactions on Vehicular Technology, vol.56, no.6, pp.3807-3817, Jun. 2007

[9] X. Sheng and Y.-H. Hu, "Maximum likelihood multiple-source localization using acoustic energy measurements with wireless sensor networks," IEEE Transactions on Signal Processing, vol.53, no.1, pp.44-53, Jan. 2005

[10] H.C. So, "Source Localization: Algorithms and Analysis," Handbook of Position Location: Theory, Practice and Advances, Chapter 2, S.A.Zekavat and M.Buehrer, Eds., Wiley-IEEE Press, 2011

[11] K.W. Cheung, H.C. So, W.-K. Ma and Y.T. Chan, "A constrained least squares approach to mobile positioning: algorithms and optimality," EURASIP Journal on Advances in Signal Processing, vol.2006, Article ID 20858, pp.1-23, 2006 
[12] A. Pages-Zamora, J. Vidal and D.H. Brooks, "Closed-form solution for positioning based on angle of arrival measurements," Proceedings of the IEEE International Symposium on Personal, Indoor and Mobile Radio Communications, vol.4, pp.15221526, Sep. 2002, Sydney, Australia

[13] P. Biswas, T.-C. Liang, T.-C. Wang and Y. Ye, "Semidefinite programming based algorithms for sensor network localization," ACM Trans. Sensor Networks, vol.2, no.2, pp.188-220, May 2006

[14] K.W.K. Lui, W.-K. Ma, H.C. So and F.K.W. Chan, "Semi-definite programming algorithms for sensor network node localization with uncertainties in anchor positions and/or propagation speed," IEEE Transactions on Signal Processing, vol.57, no.2, pp.752-763, Feb. 2009

[15] K. Yang, G. Wang and Z. Luo, "Efficient convex relaxation methods for robust target localization by a sensor network using time differences of arrivals," IEEE Transactions on Signal Processing, vol.57, no.7, pp.2775-2784, Jul. 2009

[16] K.C. Ho, X. Lu and L. Kovavisaruch, "Source localization using TDOA and FDOA measurements in the presence of receiver location errors: analysis and solution,” IEEE Transactions on Signal Processing, vol.55, no.2, pp.684-696, Feb. 2007

[17] M. Sun and K.C. Ho, "An asymptotically efficient estimator for TDOA and FDOA positioning of multiple disjoint sources in the presence of sensor location uncertainties," IEEE Transactions on Signal Processing, vol.59, no.7, pp.3434-3440, Jul. 2011

[18] H.C. So, Y.T. Chan and F.K.W. Chan, "Closed-form formulae for optimum time difference of arrival based localization," IEEE Transactions on Signal Processing, vol.56, no.6, pp.2614-2620, Jun. 2008

[19] J. Löfberg, "YALMIP : a toolbox for modeling and optimization in MATLAB," Proc. Int. Symp. CACSD, pp.284-289, Taipei, Taiwan, Sept. 2004

[20] K.C. Toh, M.J. Todd and R.H. Tutuncu, "SDPT3 - a MATLAB software package for semidefinite programming," Optimization Methods and Software, vol.11, pp.545-581, 1999

[21] R.H. Tutuncu, K.C. Toh and M.J. Todd, "Solving semidefinite-quadratic-linear programs using SDPT3," Mathematical Programming Ser. B, vol.95, pp.189-217, 2003, http://www.math.nus.edu.sg/ mattohkc/sdpt3.html 


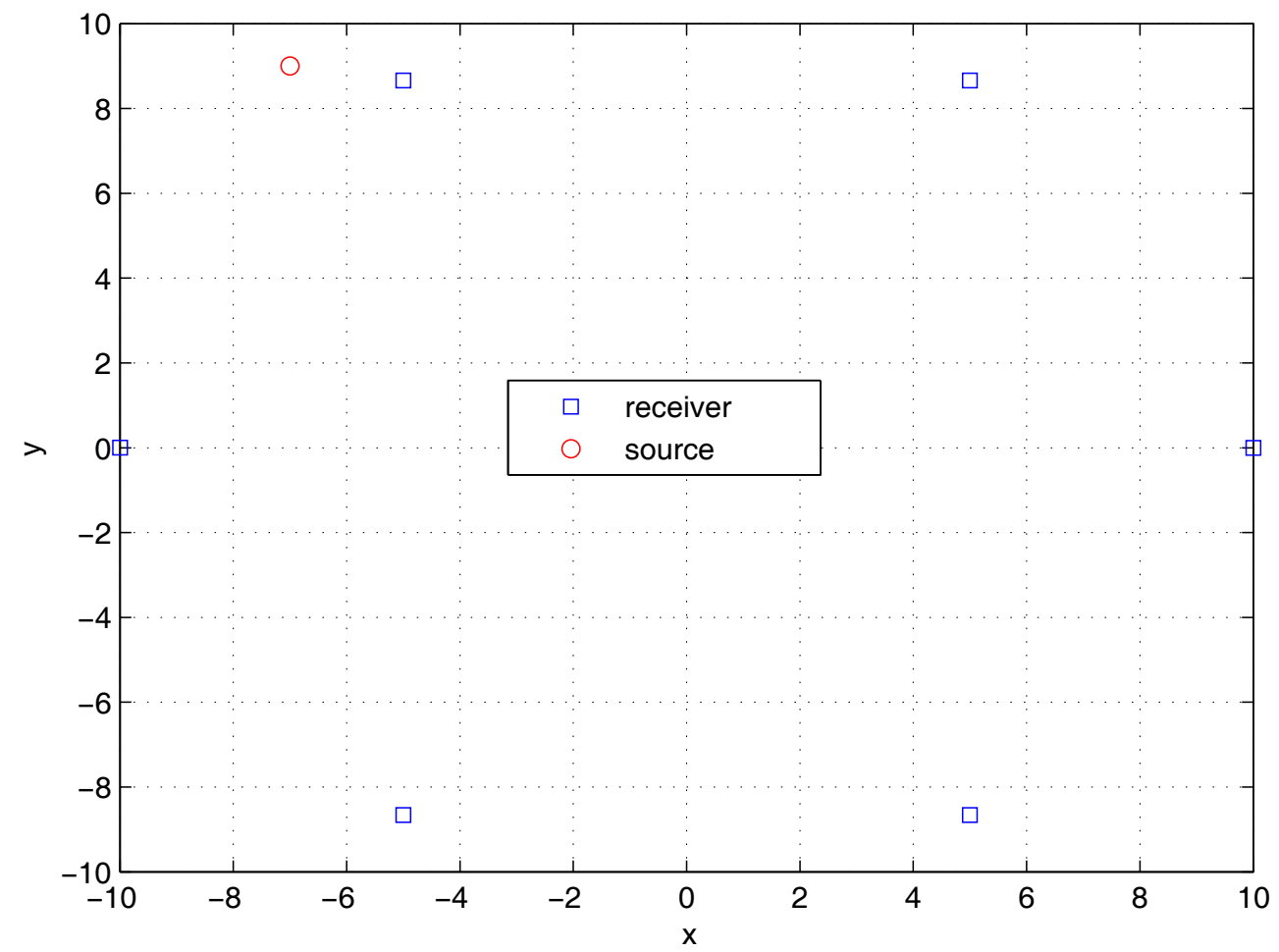

Fig. 1. Geometry for the first test 


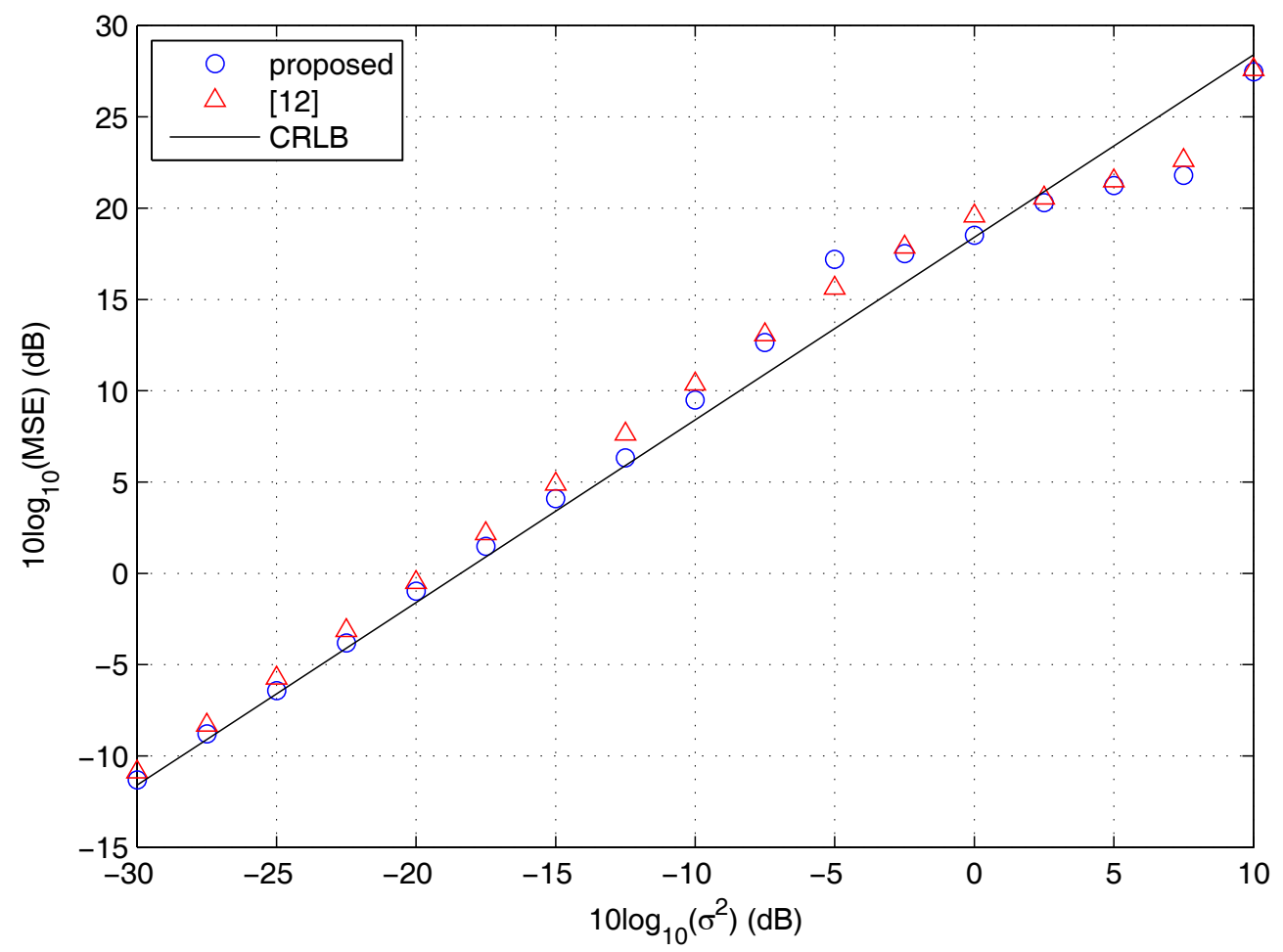

Fig. 2. Mean square error versus $\sigma^{2}$ for AOA-based positioning 


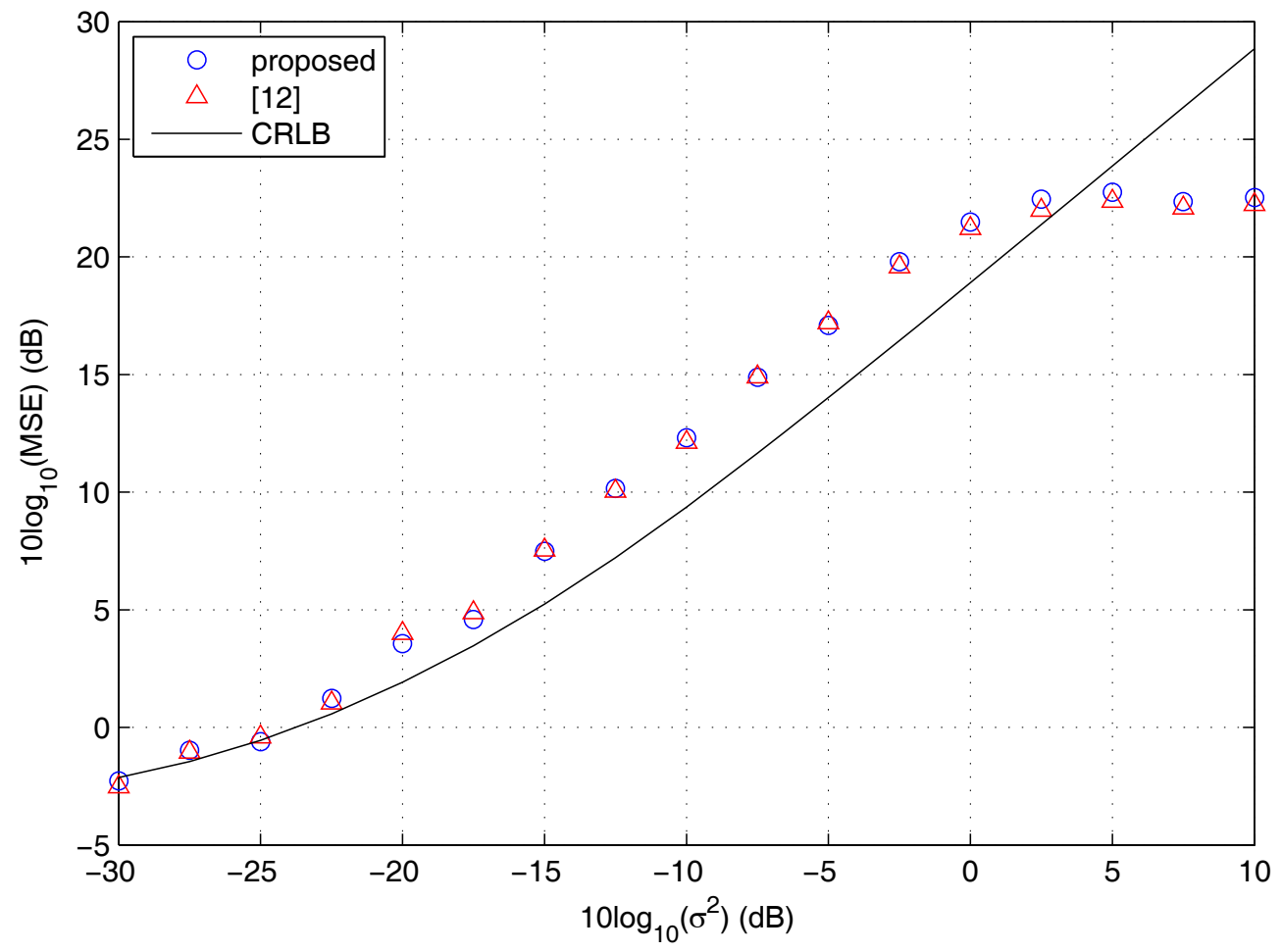

Fig. 3. Mean square error versus $\sigma^{2}$ for AOA-based positioning with receiver position uncertainty 


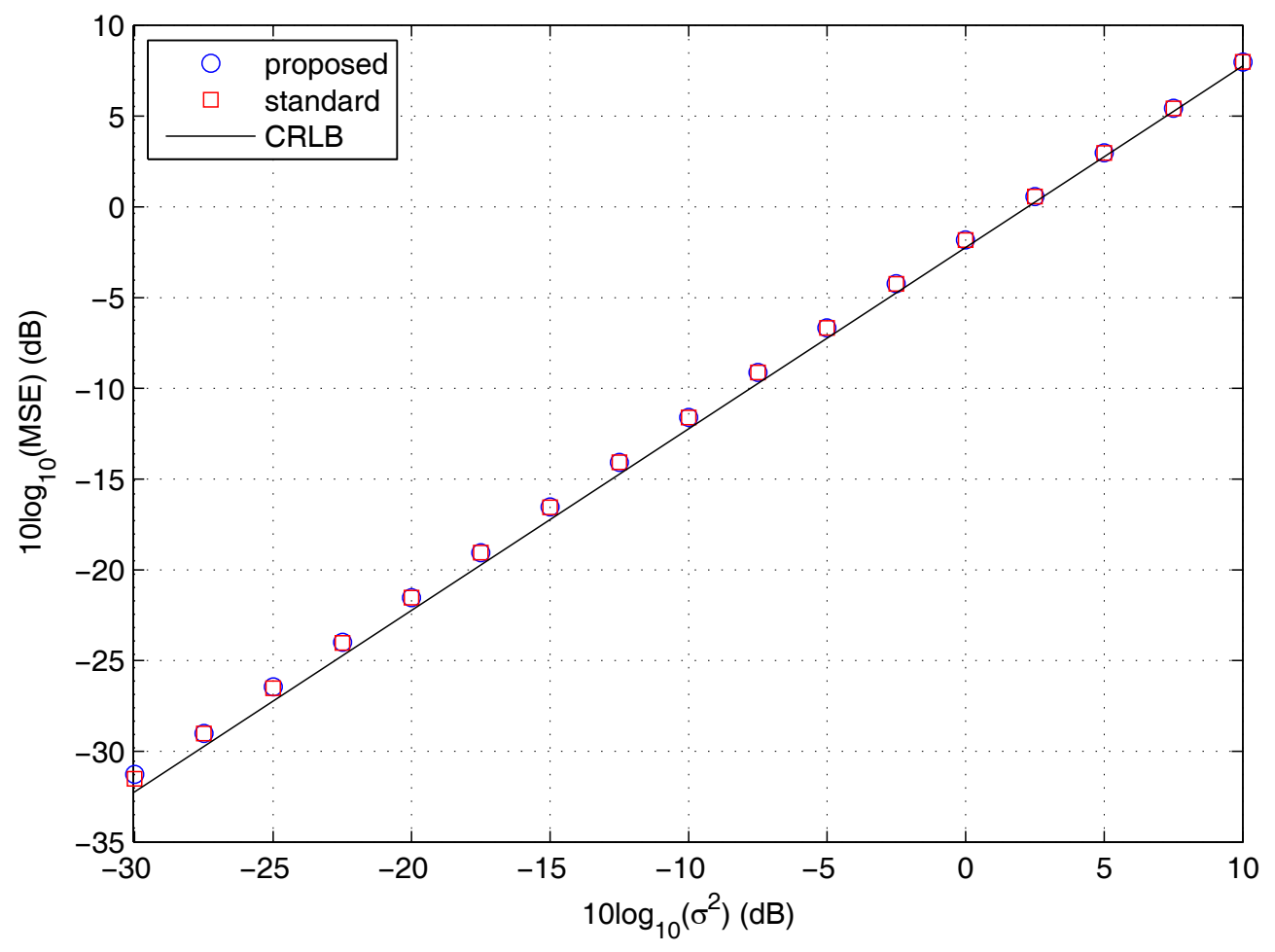

Fig. 4. Mean square error versus $\sigma^{2}$ for TOA-based positioning 


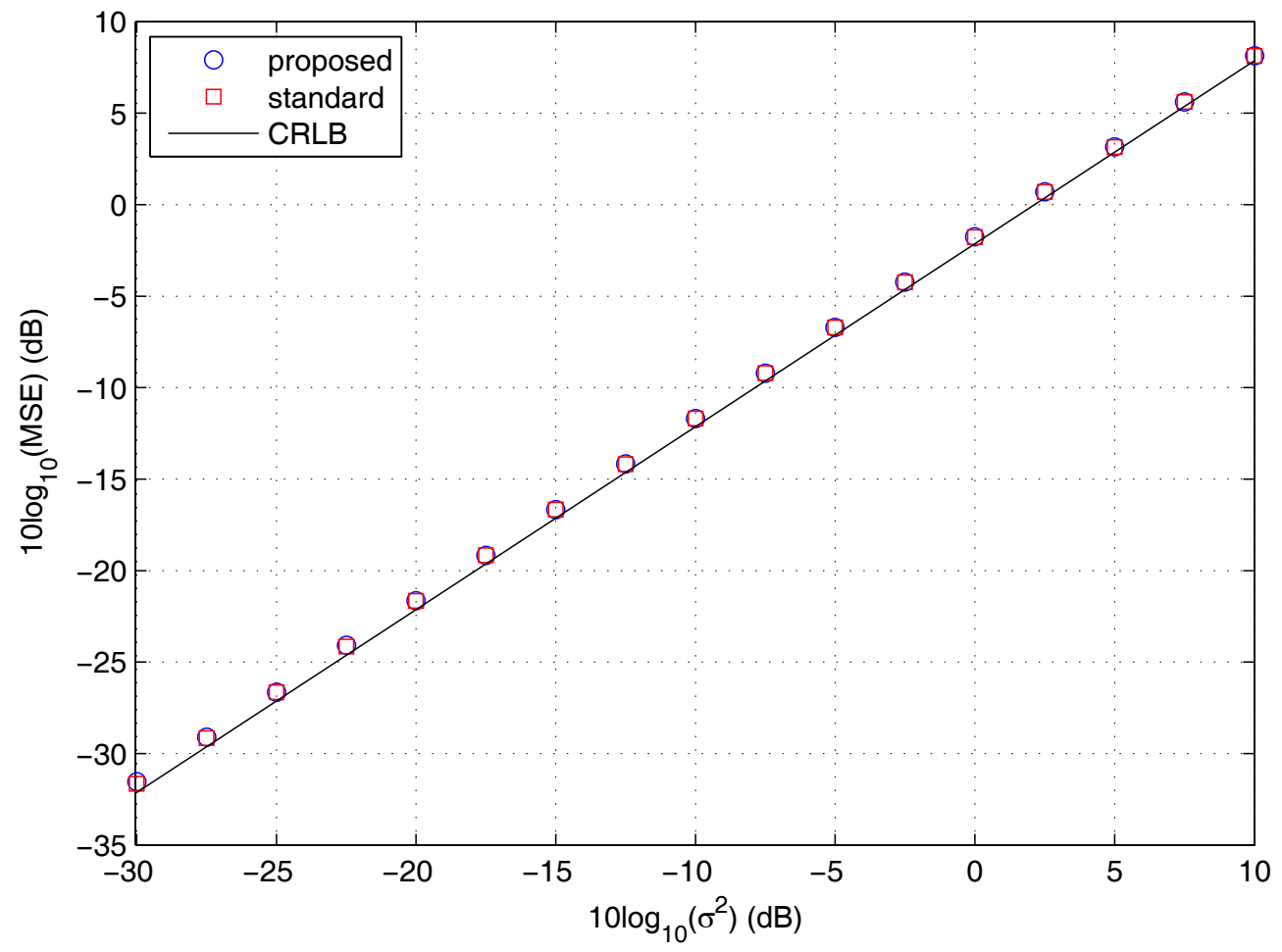

Fig. 5. Mean square error versus $\sigma^{2}$ for TOA-based positioning with a random source location 


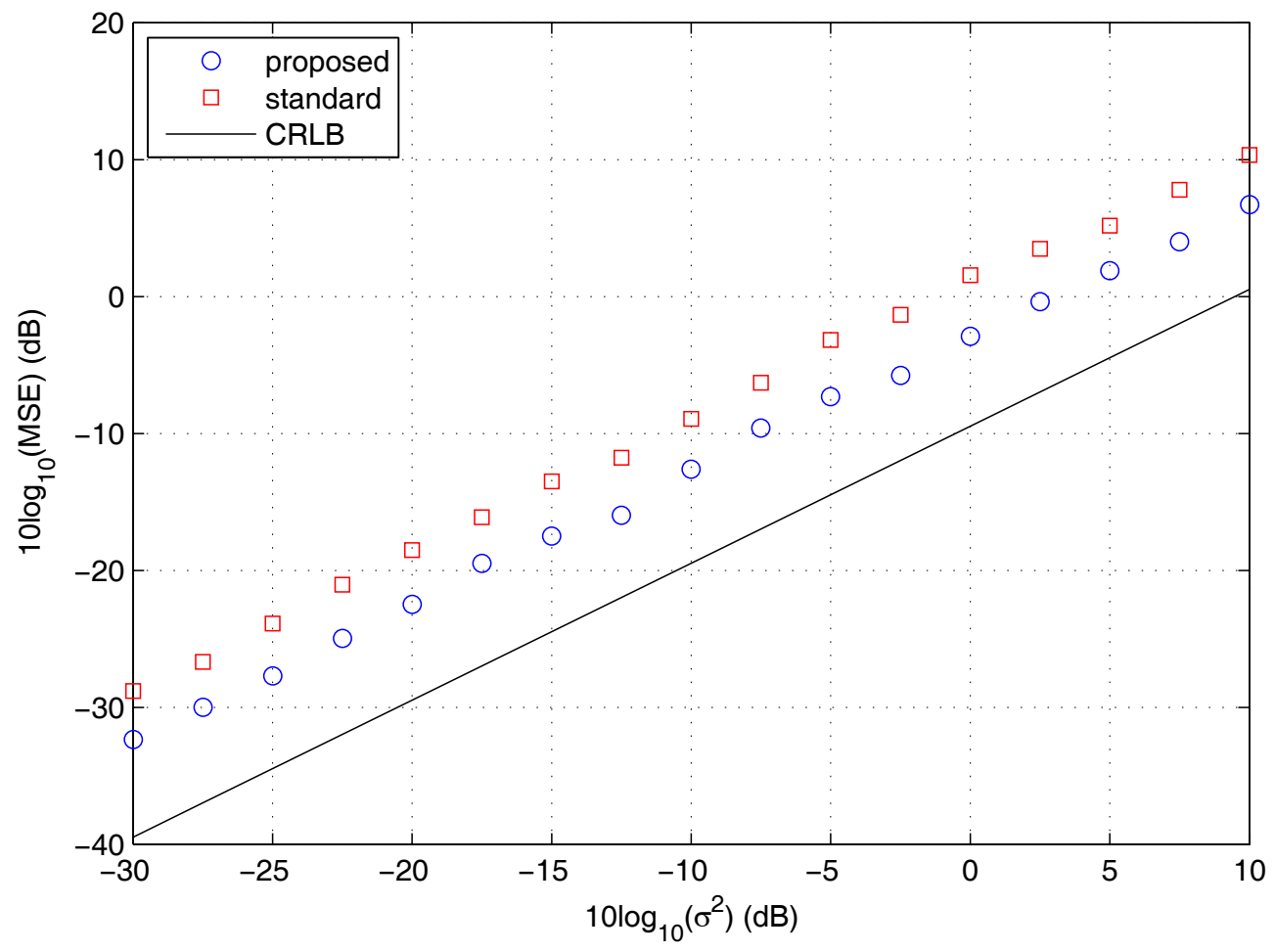

Fig. 6. Mean square error versus $\sigma^{2}$ for TOA-based positioning in colored noise 


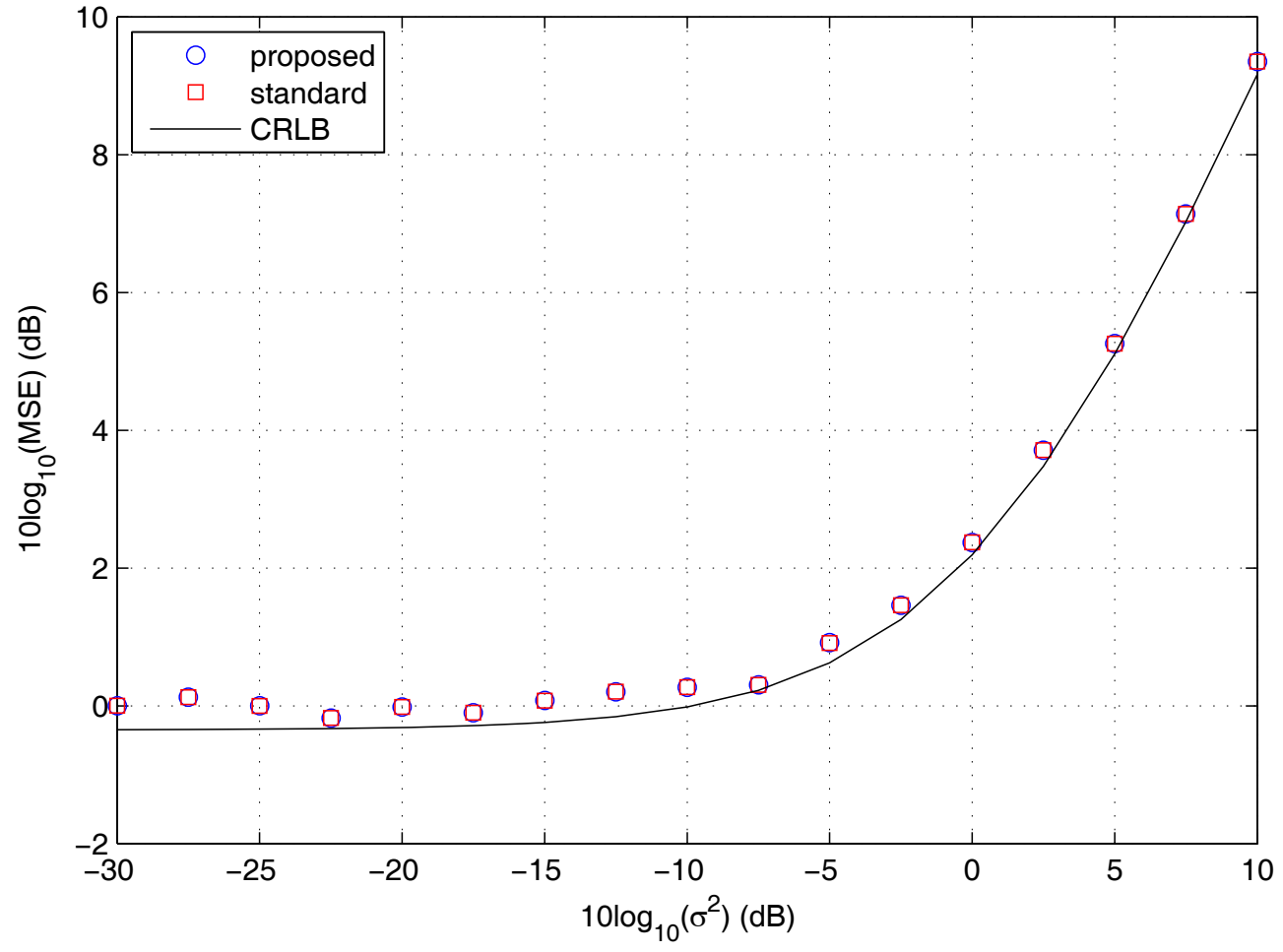

Fig. 7. Mean square error versus $\sigma^{2}$ for TOA-based positioning with receiver position uncertainty 


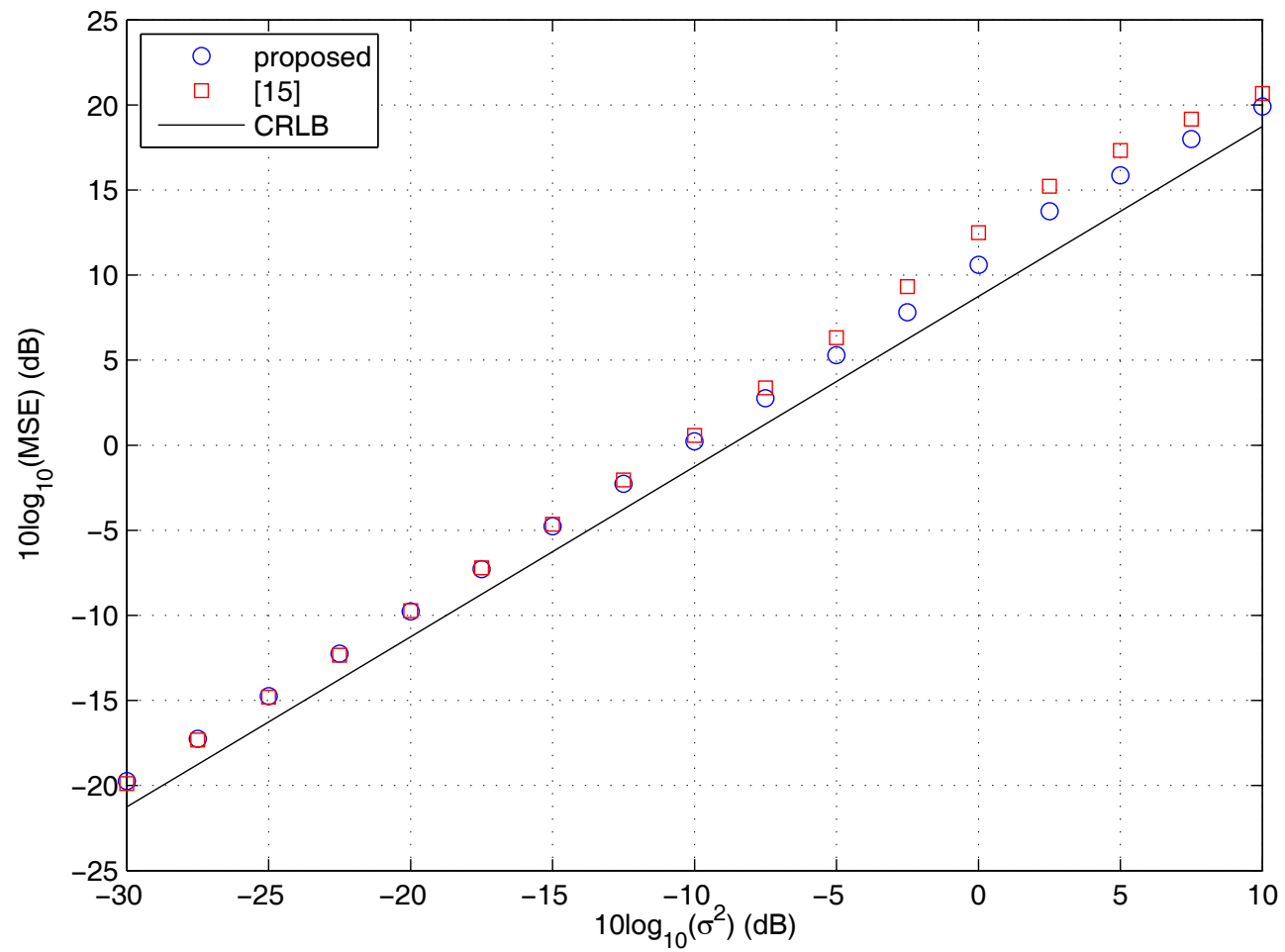

Fig. 8. Mean square error versus $\sigma^{2}$ for TDOA-based positioning 


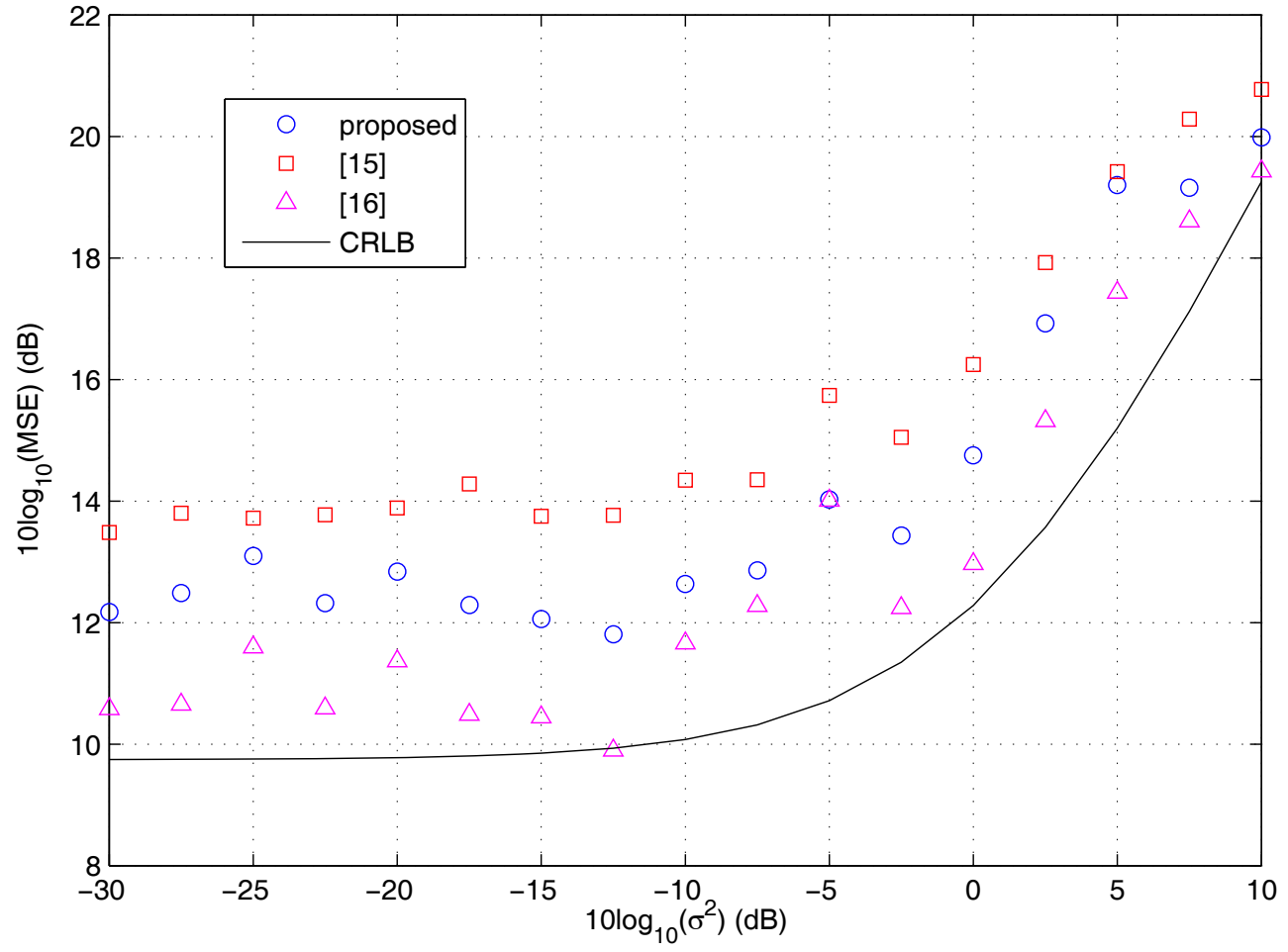

Fig. 9. Mean square error versus $\sigma^{2}$ for TDOA-based positioning with receiver position uncertainty 